\title{
Article \\ A Comprehensive Analysis of Hermite-Hadamard Type Inequalities via Generalized Preinvex Functions
}

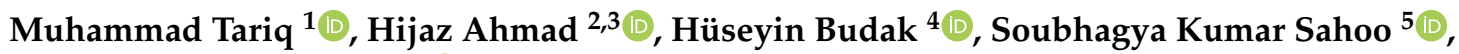 \\ Thanin Sitthiwirattham ${ }^{6, *} \mathbb{D}$ and Jiraporn Reunsumrit ${ }^{7}$ \\ 1 Department of Basic Sciences and Related Studies, Mehran University of Engineering and Technology, \\ Jamshoro 76062, Pakistan; captaintariq2187@gmail.com \\ 2 Department of Computer Engineering, Biruni University, Istanbul 34025, Turkey; hijaz555@gmail.com \\ 3 Section of Mathematics, International Telematic University Uninettuno, Corso Vittorio Emanuele II, 39, \\ 00186 Roma, Italy \\ 4 Department of Mathematics, Faculty of Science and Arts, Düzce University, Düzce 81620, Turkey; \\ hsyn.budak@gmail.com \\ 5 Department of Mathematics, Institute of Technical Education and Research, Siksha 'O' Anusandhan \\ University, Bhubaneswar 751030, India; soubhagyakumarsahoo@soa.ac.in \\ 6 Department of Mathematics, Faculty of Science and Technology, Suan Dusit University, \\ Bangkok 10300, Thailand \\ 7 Department of Mathematics, Faculty of Applied Science, King Mongkut's University of Technology \\ North Bangkok, Bangkok 10800, Thailand; jiraporn.r@sci.kmutnb.ac.th \\ * Correspondence: thanin_sit@dusit.ac.th
}

check for updates

Citation: Tariq, M.; Ahmad, H.; Budak, H.; Sahoo, S.K.;

Sitthiwirattham, T.; Reunsumrit, J. A

Comprehensive Analysis of

Hermite-Hadamard Type

Inequalities via Generalized Preinvex

Functions. Axioms 2021, 10, 328.

https://doi.org/10.3390/axioms

10040328

Academic Editor: Delfim F. M. Torres

Received: 5 September 2021

Accepted: 25 November 2021

Published: 30 November 2021

Publisher's Note: MDPI stays neutral with regard to jurisdictional claims in published maps and institutional affiliations.

Copyright: (C) 2021 by the authors. Licensee MDPI, Basel, Switzerland. This article is an open access article distributed under the terms and conditions of the Creative Commons Attribution (CC BY) license (https:/ / creativecommons.org/licenses/by/ $4.0 /)$.

\begin{abstract}
The principal objective of this article is to introduce the idea of a new class of $n$-polynomial convex functions which we call $n$-polynomial $s$-type $m$-preinvex function. We establish a new variant of the well-known Hermite-Hadamard inequality in the mode of the newly introduced concept. To add more insight into the newly introduced concept, we have discussed some algebraic properties and examples as well. Besides, we discuss a few new exceptional cases for the derived results, which make us realize that the results of this paper are the speculations and expansions of some recently known outcomes. The immeasurable concepts and chasmic tools of this paper may invigorate and revitalize additional research in this mesmerizing and absorbing field.
\end{abstract}

Keywords: $s$-type convex function; preinvex function; $m$-preinvex function; Hölder's inequality; Hölder-Íscan inequality; power-mean integral inequality; improved power-mean integral inequality

\section{Introduction}

The term convexity is one of the most elegant, natural and fundamental notations in mathematics, and for the first time in the year 1934, it was used widely in the classical and tremendous book Inequalities, which is written by great mathematicians Hardy, Littlewood and Pólya [1]. The theory of convexity presents a very impressive, remarkable and mesmerizing field of research activities. This theory has a large number of potential applications in many absorbing and worthy fields of research. Furthermore, this theory also plays a magnificent role in many different areas, such as information theory, coding theory, optimization, engineering and inequality theory. Mathematicians have proposed several new notions of classical convexity in the literature (see [2-8]) and focused on the concept of convex functions, generalizing its variant forms in different ways using innovative ideas and fruitful techniques. In this aspect, integral inequalities have performed a vital role in defining real-world problems. In this setting, Hermite-Hadamard [9] type inequalities are influential in convexity theory.

Theories of inequality along with convexity properties play an essential part in the present-day mathematical investigation. In literature there exists several well-known mathematical inequalities, but the Hermite-Hadamard inequality is considered the most and 
holds a special place in the subject of convex analysis. This inequality asserts that the mean value of the integral of the convex function on an interval, say $[a, b]$, interpolates between the mapping of the arithmetic means of the limit points of the interval and the arithmetic mean of the mappings of the limits of the interval $[a, b]$. This inequality uncovers the connection among the integral mean of a convex function, mean of mappings and mapping of the arithmetic mean of limits of the integration. A large number of research articles have been written during the last decade by several mathematicians who give new proof, generalizations, extensions and refinements of this inequality. The subject inequalities have a lot of investigations and applications in the variability of applied science, for example, coding theory, geometric function theory, impulsive diffusion equations, numerical analysis and fractional calculus. For the attention of the readers, see the references [10-12].

In 1985, G. Toader [13] for the first time explored the new family of convex functions, namely $m$-convex functions. After the contribution of Toader, Latif [14] and Kalsoom [15] polished the idea of preinvexity and explored the new notion of preinvexity namely $m-$ preinvexity. Recently, Toplu [16] investigated an n-polynomial convexity and established a new version of Hermite-Hadamard type inequality for the newly introduced function. Noor and his team then generalized the concept of $n$-polynomial convexity to $n$-polynomial harmonic convexity. Moreover, some refinements of this class of convexity are being widely studied by Tariq and his collaborators for $n$-polynomial exponentially convex function. Now, we list some of their work on $n$-polynomial convexity for references, where they introduced n-polynomial exponentially convex function [17], generalized harmonic exponential convex function [18], generalized exponential type convex function [19], studied some of algebraic properties and presented a few new kinds of Hermite-Hadamard type inequalities. Very recently, İşcan [20] in his paper introduced a new function, namely an $n$-polynomial $s-$ type convex function. Park et al. [21] and Chen et al. [22] investigated n-polynomial pconvexity and established some related integral inequalities. Awan et al. [23] generalized the harmonic convex function to n-polynomial harmonically convex function and presented related Hermite-Hadamard inequalities.

The aim of this paper is to introduce a new class of $n$-polynomial preinvex function called $n$-polynomial $s$-type $m$-preinvex function. We elaborate on some algebraic properties and examples in the manner of the newly introduced definition. The new version of Hermite-Hadamard and its refinements in the support of this newly introduced concept are investigated.

\section{Preliminaries}

Now, we present some known concepts which have helped us extensively throughout the paper to establish our main results.

Definition 1 ([24]). A set $\mathbb{X} \subseteq \mathbb{R}^{n}$ is said to be m-invex w.r.t $\eta: \mathbb{X} \times \mathbb{X} \times(0,1] \rightarrow \mathbb{R}^{n}$, if

$$
m \mu_{2}+\theta \eta\left(\mu_{1}, \mu_{2}, m\right) \in \mathbb{X}
$$

holds for every $\mu_{1}, \mu_{2} \in \mathbb{X}, m \in(0,1]$ and $\theta \in[0,1]$.

Example 1 ([24]). Let $m=\frac{1}{4}$ and $\mathbb{X}=\left[\frac{-\pi}{2}, 0\right) \cup\left(0, \frac{1}{2}\right]$

$$
\eta\left(\mu_{2}, \mu_{1}, m\right)=\left\{\begin{array}{l}
m \cos \left(\mu_{2}-\mu_{1}\right) \quad \text { if } \mu_{1} \in\left(0, \frac{\pi}{2}\right], \mu_{2} \in\left(0, \frac{\pi}{2}\right] ; \\
-m \cos \left(\mu_{2}-\mu_{1}\right) \quad \text { if } \mu_{1} \in\left[\frac{-\pi}{2}, 0\right), \mu_{2} \in\left[\frac{-\pi}{2}, 0\right) ; \\
m \cos \left(\mu_{1}\right) \quad \text { if } \mu_{1} \in\left(0, \frac{\pi}{2}\right], \mu_{2} \in\left[\frac{-\pi}{2}, 0\right) ; \\
-m \cos \left(\mu_{1}\right) \quad \text { if } \mu_{1} \in\left[-\frac{\pi}{2}, 0\right), \mu_{2} \in\left(0, \frac{\pi}{2}\right] .
\end{array}\right.
$$

Then, the above set $\mathbb{X}$ for all the mentioned conditions $\theta \in[0,1]$ is an m-invex set but not convex set. 
In the year 1988, mathematicians Weir and Mond [25] used the idea of invex set and explored the idea of preinvexity.

Definition 2 ([25]). Suppose $\mathbb{X} \neq \varnothing \in \mathbb{R}$ be an invex set w.r.t $\psi: \mathbb{X} \times \mathbb{X} \neq \varnothing \rightarrow \mathbb{R}$. Then, $\psi: \mathbb{X} \rightarrow \mathbb{R}$ is known as preinvex w.r.t $\eta$ if

$$
\psi\left(\mu_{2}+\theta \eta\left(\mu_{1}, \mu_{2}\right)\right) \leq(\theta) \psi\left(\mu_{1}\right)+(1-\theta) \psi\left(\mu_{2}\right), \quad \forall \mu_{1}, \mu_{2} \in \mathbb{X}, \theta \in[0,1] .
$$

Later on, several mathematicians studied and collaborated their thoughts on the concept of preinvexity. For some related works, we refer the readers to see the published articles [26-29].

From the last current decade, several mathematicians improved and worked on the concept of preinvex function in variant ways. Recently, Kalsoom [15] investigated and explored a generalized version of preinvexity i.e., generalized $m$-preinvex function, which is stated as:

Definition 3. Suppose $\psi: \mathbb{X} \rightarrow \mathbb{R} \psi$ is said to be a generalized m-preinvex with respect to $\eta: \mathbb{X} \times \mathbb{X}$ $\times(0,1] \rightarrow \mathbb{R}^{n}$ for fixed $m \in(0,1]$, if

$$
\psi\left(m \mu_{2}+\theta \eta\left(\mu_{1}, \mu_{2}, m\right)\right) \leq \theta \psi\left(\mu_{1}\right)+m(1-\theta) \psi\left(\mu_{2}\right),
$$

holds for every $\mu_{1}, \mu_{2} \in \mathbb{X}, \theta \in[0,1]$.

Definition 4 ([20]). A non-negative function $\psi: \mathbb{X} \rightarrow \mathbb{R}$ is said to be s-type convex function if $\forall$ $\mu_{1}, \mu_{2} \in I, n \in \mathbb{N}, s \in[0,1]$ and $\theta \in[0,1]$, if

$$
\psi\left(\theta \mu_{1}+(1-\theta) \mu_{2}\right) \leq[1-(s(1-\theta))] \psi\left(\mu_{1}\right)+[1-s \theta] \psi\left(\mu_{2}\right) .
$$

Definition 5 ([16]). A non-negative real-valued function $\psi: \mathbb{X} \rightarrow \mathbb{R}$ is known as n-polynomial convex function, if

$$
\psi\left(\theta \mu_{1}+(1-\theta) \mu_{2}\right) \leq \frac{1}{n} \sum_{i=1}^{n}\left[1-(1-\theta)^{i}\right] \psi\left(\mu_{1}\right)+\frac{1}{n} \sum_{i=1}^{n}\left[1-\theta^{i}\right] \psi\left(\mu_{2}\right),
$$

holds for every $\mu_{1}, \mu_{2} \in \mathbb{X}, \theta \in[0,1], s \in[0,1]$ and $n \in \mathbb{N}$.

Mohan and Neogy [30] gave the following hypothesis which is widely used in the concept of preinvexity.

Condition-C: Let $\mathbb{X} \subset R^{n}$ be an open invex subset with respect to $\eta: \mathbb{X} \times \mathbb{X} \rightarrow \mathbb{R}$. For any $\mu_{1}, \mu_{2} \in \mathbb{X}$ and $\theta \in[0,1]$,

$$
\begin{array}{r}
\eta\left(\mu_{2}, \mu_{2}+\theta \eta\left(\mu_{1}, \mu_{2}\right)\right)=-\theta \eta\left(\mu_{1}, \mu_{2}\right) . \\
\eta\left(\mu_{1}, \mu_{2}+\theta \eta\left(\mu_{1}, \mu_{2}\right)\right)=(1-\theta) \eta\left(\mu_{1}, \mu_{2}\right) .
\end{array}
$$

For any $\mu_{1}, \mu_{2} \in \mathbb{X}$ and $\theta_{1}, \theta_{2} \in[0,1]$ from Condition $C$, we have

$$
\eta\left(\mu_{2}+\theta_{2} \eta\left(\mu_{1}, \mu_{2}\right), \mu_{2}+\theta_{1} \eta\left(\mu_{1}, \mu_{2}\right)\right)=\left(\theta_{2}-\theta_{1}\right) \eta\left(\mu_{1}, \mu_{2}\right) .
$$

The above Condition $C$ is also presented by the name of extended Condition $C$ in the sense of $m$-preinvexity by Ting Song Du [31]. 
Extended Condition-C: Let $\mathbb{X} \subset \mathbb{R}^{n}$ be an open $m$-invex subset with respect to $\eta: \mathbb{X} \times \mathbb{X}$ $\times(0,1] \rightarrow \mathbb{R}$, for any $\mu_{1}, \mu_{2} \in \mathbb{X}, \theta \in[0,1]$. We then have

$$
\begin{array}{r}
\eta\left(\mu_{2}, m \mu_{2}+\theta \eta\left(\mu_{1}, \mu_{2}, m\right), m\right)=-\theta \eta\left(\mu_{1}, \mu_{2}, m\right) \\
\eta\left(\mu_{1}, m \mu_{2}+\theta \eta\left(\mu_{1}, \mu_{2}, m\right), m\right)=(1-\theta) \eta\left(\mu_{1}, \mu_{2}, m\right) \\
\eta\left(\mu_{1}, \mu_{2}, m\right)=-\eta\left(\mu_{2}, \mu_{1}, m\right) .
\end{array}
$$

Definition 6 ([3]). Suppose $\forall \mu_{1}, \mu_{2} \in \mathbb{R}$, then an inequality of the form

$$
\left(\psi\left(\mu_{1}\right)-\psi\left(\mu_{2}\right)\right)\left(\varphi\left(\mu_{1}\right)-\varphi\left(\mu_{2}\right)\right) \geq 0
$$

is known as similarly ordered.

Studying the above concepts and energized by the ongoing research activities, the article is structured as follows. In Section 3, we discuss the notion of $n$-polynomial s-type $m$-preinvexity and studied related properties. Section 4 deals with establishing a new sort of Hermite-Hadamard type inequality. In Section 5, two new integral identities are established, based on these identities and with the help of the new notion of preinvexity, some refinements and estimations of Hermite-Hadamard type inequality are given. In the last Section 6, a brief conclusion and the future scopes are discussed.

\section{Generalized Preinvex Function and Its Properties}

Here, we are going to elaborate the new notion for convex functions which is called generalized preinvex function, i.e., $n$-polynomial $s$-type $m$-preinvex function, and study some of its algebraic properties and examples.

Definition 7. Suppose $\mathbb{X} \subset \mathbb{R}$ be a nonempty m-invex set w.r.t $\eta: \mathbb{X} \times \mathbb{X} \times(0,1] \rightarrow \mathbb{R}$. Then, $\psi: \mathbb{X} \rightarrow \mathbb{R}$ is known as n-polynomial s-type m-preinvex if

$$
\psi\left(m \mu_{2}+\theta \eta\left(\mu_{1}, \mu_{2}, m\right)\right) \leq \frac{1}{n} \sum_{i=1}^{n}(1-(s(1-\theta)))^{i} \psi\left(\mu_{1}\right)+\frac{1}{n} \sum_{i=1}^{n} m^{i}\left(1-(s \theta)^{i}\right) \psi\left(\mu_{2}\right),
$$

holds for every $\mu_{1}, \mu_{2} \in \mathbb{X}, m \in(0,1], s \in[0,1], \theta \in[0,1]$ and $n \in \mathbb{N}$.

Remark 1. (i) If $n=1$, then

$$
\psi\left(m \mu_{2}+\theta \eta\left(\mu_{1}, \mu_{2}, m\right)\right) \leq(1-(s(1-\theta))) \psi\left(\mu_{1}\right)+m(1-s \theta) \psi\left(\mu_{2}\right) .
$$

(ii) If $n=s=m=1$, then Definition 7 is directly reduced to the definition of preinvexity which was explored by Weir et al. [25].

(iii) If $n=m=1$ and $\eta\left(\mu_{1}, \mu_{2}, m\right)=\mu_{1}-m \mu_{2}$, then Definition 7 is directly reduced to the s-type convex function which was explored by İscan et al. [20].

(iv) If $n=s=m=1$ and $\eta\left(\mu_{1}, \mu_{2}, m\right)=\mu_{1}-m \mu_{2}$, then Definition 7 is directly reduced to definition of convex function which was investigated by Niculescu et al. [3].

Lemma 1. Suppose $\forall \theta \in[0,1], m \in(0,1], n \in \mathbb{N}$ and $s \in[0,1]$, then the following inequalities

$$
\theta \leq \frac{1}{n} \sum_{i=1}^{n}(1-(s(1-\theta)))^{i} \text { and } m(1-\theta) \leq \frac{1}{n} \sum_{i=1}^{n} m^{i}\left(1-(s \theta)^{i}\right)
$$

are hold.

Proof. The proof is obvious. 
Lemma 2. Suppose $\forall \theta \in[0,1], m \in(0,1], n \in \mathbb{N}$ and $s \in[0,1]$, then the following inequalities

$$
(1-(s(1-\theta))) \leq \frac{1}{n} \sum_{i=1}^{n}(1-(s(1-\theta)))^{i} \quad \text { and } \quad m(1-s \theta) \leq \frac{1}{n} \sum_{i=1}^{n} m^{i}\left(1-(s \theta)^{i}\right)
$$

are hold.

Proof. The proof is obvious.

Proposition 1. Suppose $\forall \theta \in[0,1], m \in(0,1], n \in \mathbb{N}$ and $s \in[0,1]$, then every non-negative $m$-preinvex function is an $n$-polynomial s-type $m$-preinvex function.

Proof. According to the property of $m$-preinvexity and Lemma 1, we have

$$
\begin{aligned}
\psi\left(m \mu_{2}+\theta \eta\left(\mu_{1}, \mu_{2}, m\right)\right) & \leq \theta \psi\left(\mu_{1}\right)+m(1-\theta) \psi\left(\mu_{2}\right) \\
& \leq \frac{1}{n} \sum_{i=1}^{n}(1-(s(1-\theta)))^{i} \psi\left(\mu_{1}\right)+\frac{1}{n} \sum_{i=1}^{n} m^{i}\left(1-(s \theta)^{i}\right) \psi\left(\mu_{2}\right) .
\end{aligned}
$$

Proposition 2. Suppose $\forall \theta \in[0,1], m \in(0,1], n \in \mathbb{N}$ and $s \in[0,1]$, then every non-negative $s$-type $m$-preinvex function is an n-polynomial s-type $m$-preinvex function.

Proof. According to the property of $s$-type $m$-preinvexity and Lemma 2 , we have

$$
\begin{aligned}
\psi\left(m \mu_{2}+\theta \eta\left(\mu_{1}, \mu_{2}, m\right)\right) & \leq(1-(s(1-\theta))) \psi\left(\mu_{1}\right)+m(1-s \theta) \psi\left(\mu_{2}\right) \\
& \leq \frac{1}{n} \sum_{i=1}^{n}(1-(s(1-\theta)))^{i} \psi\left(\mu_{1}\right)+\frac{1}{n} \sum_{i=1}^{n} m^{i}\left(1-(s \theta)^{i}\right) \psi\left(\mu_{2}\right) .
\end{aligned}
$$

Proposition 3. Every non-negative n-polynomial s-type $m$-preinvex function for $s \in[0,1], m \in(0$, $1], n \in \mathbb{N}$, and $\theta \in[0,1]$ is an $(h, m)$-preinvex function with $h(\theta)=\frac{1}{n} \sum_{i=1}^{n}(1-(s(1-\theta)))^{i}$.

Proof. Using the definition of $n$-polynomial $s$-type $m$-preinvexity for $s \in[0,1], m \in(0,1]$, $\theta \in[0,1]$ and the condition for $h(\theta)=\frac{1}{n} \sum_{i=1}^{n}(1-(s(1-\theta)))^{i}$, we have

$$
\begin{aligned}
\psi\left(m \mu_{2}+\theta \eta\left(\mu_{1}, \mu_{2}, m\right)\right) & \leq \frac{1}{n} \sum_{i=1}^{n}(1-(s(1-\theta)))^{i} \psi\left(\mu_{1}\right)+\frac{1}{n} \sum_{i=1}^{n} m^{i}\left(1-(s \theta)^{i}\right) \psi\left(\mu_{2}\right) \\
& \leq h(\theta) \psi\left(\mu_{1}\right)+m h(1-\theta) \psi\left(\mu_{2}\right)
\end{aligned}
$$

From the above proposition, one can easily observe that the newly defined preinvexity is very large concerning the previously known functions, such as preinvex functions and convex functions. This is the most beautiful aspect of the proposed new Definition 7.

Now, we demonstrate examples in support of the new idea. 
Example 2. $\psi(\mu)=|\mu| \quad \forall \mu \geq 0$ is a non-negative convex function, but every convex is a preinvex function (see [32]). Thus, due to this reason, it is a non-negative m-preinvex function if $m=1$. By using Proposition 1, it is an n-polynomial s-type m-preinvex function.

Example 3. $\psi(\mu)=e^{\mu} \quad \forall \mu \geq 0$ is a non-negative convex function, but every convex is preinvex function (see [32]). For this reason, it is non-negative m-preinvex function if $m=1$. By using Proposition 1, it is an n-polynomial s-type m-preinvex function.

Now, we explore and elaborate some examples regarding the newly introduced idea. We can see that $\psi(x)=-|x|$ is preinvex but not convex.

Thus, next we will present some examples for the introduced $n$-polynomial $s$-type $m$-preinvex function w.r.t $\eta$ on $\mathbb{X}$, but it is a non-negative and not convex function.

Example 4. Suppose $\psi(x): \mathbb{R}^{+} \rightarrow \mathbb{R}^{+}$be defined by

$\psi(\mu)= \begin{cases}\mu & ; 0 \leq \mu \leq 1 \\ 1 & ; \mu>1\end{cases}$

and

$\eta\left(\mu_{1}, \mu_{2}, m\right)= \begin{cases}\mu_{1}-m \mu_{2} & ; \mu_{1} \leq 0, \mu_{2} \leq 0 \\ \mu_{1}-m \mu_{2} & ; 0 \leq \mu_{1} \leq 1, \mu_{2} \leq 1 \\ -2-m \mu_{2} & ; \mu_{1} \leq 0,0 \leq \mu_{2} \leq 1 \\ 2-m \mu_{2} & ; 0 \leq \mu_{1} \leq 1, \mu_{2} \leq 0\end{cases}$

The non-negative function $\psi(x)$ is m-preinvex but not convex. According to Proposition 1, it is an $n$-polynomial s-type $m$-preinvex function with respect to $\eta$ on $X$ if $m=1$.

Example 5. Let $\psi(x): \mathbb{R}^{+} \rightarrow \mathbb{R}^{+}$be defined by

$\psi(\mu)=\left\{\begin{array}{l}\mu+1 \quad ; 0 \leq \mu \leq 1 \\ 1 \quad ; \mu>1\end{array}\right.$

and

$\eta\left(\mu_{1}, \mu_{2}, m\right)=\left\{\begin{array}{l}\mu_{1}+m \mu_{2} ; \mu_{1} \leq \mu_{2} \\ 2\left(\mu_{1}+m \mu_{2}\right) ; \mu_{1}>\mu_{2}\end{array}\right.$

$\forall \mu_{1}, \mu_{2} \in \mathbb{R}^{+}=[0,+\infty)$. The above non-negative function $\psi(x)$ is m-preinvex but not convex. According to Proposition 1, it is an n-polynomial s-type preinvex function if $m=1$.

Theorem 1. Let $\psi, \varphi: \mathbb{X}=\left[\mu_{1}, \mu_{2}\right] \rightarrow \mathbb{R}$. If $\psi, \varphi$ are two $n$-polynomial s-type m-preinvex functions, then

(i) $(\psi+\varphi)$ is also an n-polynomial s-type m-preinvex function.

(ii) $(c \psi)$ is also an $n$-polynomial s-type m-preinvex function.

Proof. (i)

$$
\begin{aligned}
& (\psi+\varphi)\left(m \mu_{2}+\theta \eta\left(\mu_{1}, \mu_{2}, m\right)\right) \\
& =\psi\left(m \mu_{2}+\theta \eta\left(\mu_{1}, \mu_{2}, m\right)\right)+\varphi\left(m \mu_{2}+\theta \eta\left(\mu_{1}, \mu_{2}, m\right)\right) \\
& \leq \frac{1}{n} \sum_{i=1}^{n}(1-(s(1-\theta)))^{i} \psi\left(\mu_{1}\right)+\frac{1}{n} \sum_{i=1}^{n} m^{i}\left(1-(s \theta)^{i}\right) \psi\left(\mu_{2}\right) \\
& +\frac{1}{n} \sum_{i=1}^{n}(1-(s(1-\theta)))^{i} \varphi\left(\mu_{1}\right)+\frac{1}{n} \sum_{i=1}^{n} m^{i}\left(1-(s \theta)^{i}\right) \varphi\left(\mu_{2}\right) \\
& =\frac{1}{n} \sum_{i=1}^{n}(1-(s(1-\theta)))^{i}\left[\psi\left(\mu_{1}\right)+\varphi\left(\mu_{1}\right)\right]+\frac{1}{n} \sum_{i=1}^{n} m^{i}\left(1-(s \theta)^{i}\right)\left[\psi\left(\mu_{2}\right)+\varphi\left(\mu_{2}\right)\right] \\
& =\frac{1}{n} \sum_{i=1}^{n}(1-(s(1-\theta)))^{i}(\psi+\varphi)\left(\mu_{1}\right)+\frac{1}{n} \sum_{i=1}^{n} m^{i}\left(1-(s \theta)^{i}\right)(\psi+\varphi)\left(\mu_{2}\right) .
\end{aligned}
$$


(ii)

$$
\begin{aligned}
& (c \psi)\left(m \mu_{2}+\theta \eta\left(\mu_{1}, \mu_{2}, m\right)\right) \\
& \leq c\left[\frac{1}{n} \sum_{i=1}^{n}(1-(s(1-\theta)))^{i} \psi\left(\mu_{1}\right)+\frac{1}{n} \sum_{i=1}^{n} m^{i}\left(1-(s \theta)^{i}\right) \psi\left(\mu_{2}\right)\right] \\
& =\frac{1}{n} \sum_{i=1}^{n}(1-(s(1-\theta)))^{i} c \psi\left(\mu_{1}\right)+\frac{1}{n} \sum_{i=1}^{n} m^{i}\left(1-(s \theta)^{i}\right) c \psi\left(\mu_{2}\right) \\
& =\frac{1}{n} \sum_{i=1}^{n}(1-(s(1-\theta)))^{i}(c \psi)\left(\mu_{1}\right)+\frac{1}{n} \sum_{i=1}^{n} m^{i}\left(1-(s \theta)^{i}\right)(c \psi)\left(\mu_{2}\right) .
\end{aligned}
$$

This is the required proof.

Theorem 2. Suppose $\forall \theta \in[0,1], m \in(0,1], n \in \mathbb{N}$ and $s \in[0,1]$. Let $\psi: \mathbb{X} \rightarrow \mathbb{Y}$ and $\varphi: \mathbb{Y} \rightarrow \mathbb{R}$ be an n-polynomial s-type m-preinvex and non-decreasing function respectively. Then, $(\varphi \circ \psi)$ is also an n-polynomial s-type m-preinvex function w.r.t same $\eta$.

\section{Proof.}

$$
\begin{aligned}
& (\varphi \circ \psi)\left(m \mu_{2}+\theta \eta\left(\mu_{1}, \mu_{2}, m\right)\right) \\
& =\varphi\left(\psi\left(m \mu_{2}+\theta \eta\left(\mu_{1}, \mu_{2}, m\right)\right)\right) \\
& \leq \varphi\left[\frac{1}{n} \sum_{i=1}^{n}(1-(s(1-\theta)))^{i} \psi\left(\mu_{1}\right)+\frac{1}{n} \sum_{i=1}^{n} m^{i}\left(1-(s \theta)^{i}\right) \psi\left(\mu_{2}\right)\right] \\
& \leq \frac{1}{n} \sum_{i=1}^{n}(1-(s(1-\theta)))^{i} \varphi\left(\psi\left(\mu_{1}\right)\right)+\frac{1}{n} \sum_{i=1}^{n} m^{i}\left(1-(s \theta)^{i}\right) \varphi\left(\psi\left(\mu_{2}\right)\right) \\
& =\frac{1}{n} \sum_{i=1}^{n}(1-(s(1-\theta)))^{i}(\varphi \circ \psi)\left(\mu_{1}\right)+\frac{1}{n} \sum_{i=1}^{n} m^{i}\left(1-(s \theta)^{i}\right)(\varphi \circ \psi)\left(\mu_{2}\right) .
\end{aligned}
$$

Remark 2. (i) Considering $n=s=1$ in the above Theorem 2, then

$$
(\varphi \circ \psi)\left(m \mu_{2}+\theta \eta\left(\mu_{1}, \mu_{2}, m\right)\right) \leq \theta(\varphi \circ \psi)\left(\mu_{1}\right)+m(1-\theta)(\varphi \circ \psi)\left(\mu_{2}\right) .
$$

(ii) Considering $n=s=m=1$ in the above Theorem 2, then

$$
(\varphi \circ \psi)\left(\mu_{2}+\theta \eta\left(\mu_{1}, \mu_{2}\right)\right) \leq \theta(\varphi \circ \psi)\left(\mu_{1}\right)+(1-\theta)(\varphi \circ \psi)\left(\mu_{2}\right) .
$$

(iii) Considering $n=m=1$ and $\eta\left(\mu_{1}, \mu_{2}, m\right)=\mu_{1}-m \mu_{2}$ in the above Theorem 2, then

$$
(\varphi \circ \psi)\left(\theta \mu_{1}+(1-\theta) \mu_{2}\right) \leq[1-(s(1-\theta))](\varphi \circ \psi)\left(\mu_{1}\right)+[1-s \theta](\varphi \circ \psi)\left(\mu_{2}\right) .
$$

Theorem 3. Let $0<\mu_{1}<\mu_{2}, \psi_{j}: \mathbb{X}=\left[\mu_{1}, \mu_{2}\right] \rightarrow[0,+\infty)$ be a family of $n$-polynomial s-type $m$-preinvex function with respect to $\eta$ and $\psi(u)=\sup _{j} \psi_{j}(u)$. Then, $\psi$ is an n-polynomial s-type $m$-preinvex function with respect to $\eta$ for $s \in[0,1], m \in(0,1], \theta \in[0,1]$, and $U=\left\{\mu \in\left[\mu_{1}\right.\right.$, $\left.\left.\mu_{2}\right]: \psi\left(\mu_{i}\right)<\infty\right\}$ is an interval. 
Proof. Let $\mu_{1}, \mu_{2} \in U, s \in[0,1], m \in(0,1]$ and $\theta \in[0,1]$, then

$$
\begin{aligned}
& \psi\left(m \mu_{2}+\theta \eta\left(\mu_{1}, \mu_{2}, m\right)\right) \\
& =\sup _{j} \psi_{j}\left(m \mu_{2}+\theta \eta\left(\mu_{1}, \mu_{2}, m\right)\right) \\
& \leq \frac{1}{n} \sum_{i=1}^{n}(1-(s(1-\theta)))^{i} \sup _{j} \psi_{j}\left(\mu_{1}\right)+\frac{1}{n} \sum_{i=1}^{n} m^{i}\left(1-(s \theta)^{i}\right) \sup _{j} \psi_{j}\left(\mu_{2}\right) \\
& =\frac{1}{n} \sum_{i=1}^{n}(1-(s(1-\theta)))^{i} \psi\left(\mu_{1}\right)+\frac{1}{n} \sum_{i=1}^{n} m^{i}\left(1-(s \theta)^{i}\right) \psi\left(\mu_{2}\right)<\infty .
\end{aligned}
$$

This is the required proof.

Theorem 4. If $\psi_{i}: \mathbb{R}^{n} \rightarrow \mathbb{R}$ is an n-polynomial s-type m-preinvex function with respect to $\eta$ for $s \in[0,1], m \in(0,1]$ and $\theta \in[0,1]$, then the set $\mathbb{M}=\left\{\mu \in \mathbb{R}: \psi_{i}(\mu) \leq 0, i=1,2,3, \ldots, n\right\}$ is an $m$-invex set.

Proof. Since $\psi_{i}(\mu),(i=1,2,3, \ldots, n)$ are $n$-polynomial s-type $m$-preinvex functions with respect to $\eta$ for $s \in[0,1], m \in(0,1]$ and $\theta \in[0,1]$, then for all $\mu_{1}, \mu_{2} \in \mathbb{R}^{n}$,

$$
\psi_{i}\left(m \mu_{2}+\theta \eta\left(\mu_{1}, \mu_{2}, m\right)\right) \leq \frac{1}{n} \sum_{i=1}^{n}(1-(s(1-\theta)))^{i} \psi\left(\mu_{1}\right)+\frac{1}{n} \sum_{i=1}^{n} m^{i}\left(1-(s \theta)^{i}\right) \psi\left(\mu_{2}\right)
$$

holds, where $i=1,2,3, \ldots, n$.

When $\mu_{1}, \mu_{2} \in \mathbb{M}$, we know $\psi_{i}\left(\mu_{1}\right) \leq 0$ and $\psi_{i}\left(\mu_{2}\right) \leq 0$, from the above inequality, which yields that

$$
\psi_{i}\left(m \mu_{2}+\theta \eta\left(\mu_{1}, \mu_{2}, m\right)\right) \leq 0, \quad i=1,2,3, \ldots, n .
$$

That is, $m \mu_{2}+\theta \eta\left(\mu_{1}, \mu_{2}, m\right) \in \mathbb{M}$. Hence, $\mathbb{M}$ is an $m$-invex set.

Theorem 5. If $\psi: \mathbb{X} \subseteq \mathbb{R}^{n} \rightarrow \mathbb{R}$ is an n-polynomial s-type m-preinvex function on m-invex set $\mathbb{X}$ w.r.t $\eta$ for $s \in[0,1], m \in(0,1]$ and $\theta \in[0,1]$, then the function $\psi$ is also generalized quasi $m$-preinvex function on $m$-invex set $\mathbb{X}$ w.r.t $\eta$.

Proof. Since $\psi$ is an $n$-polynomial $s$-type $m$-preinvex function w.r.t $\eta$ for $s \in[0,1], m \in$ $(0,1]$ and $\theta \in[0,1]$, and we assume that $\psi\left(\mu_{1}\right) \leq \psi\left(\mu_{2}\right)$, then for all $\mu_{1}, \mu_{2} \in \mathbb{X}$, we have

$$
\begin{aligned}
& \psi\left(m \mu_{2}+\theta \eta\left(\mu_{1}, \mu_{2}, m\right)\right) \\
& \leq \frac{1}{n} \sum_{i=1}^{n}(1-(s(1-\theta)))^{i} \psi\left(\mu_{1}\right)+\frac{1}{n} \sum_{i=1}^{n} m^{i}\left(1-(s \theta)^{i}\right) \psi\left(\mu_{2}\right) \\
& \leq\left[\frac{1}{n} \sum_{i=1}^{n}(1-(s(1-\theta)))^{i}+\frac{1}{n} \sum_{i=1}^{n} m^{i}\left(1-(s \theta)^{i}\right)\right] \psi\left(\mu_{2}\right) \\
& \leq \psi\left(\mu_{2}\right) .
\end{aligned}
$$

In the same manner, if we let $\psi\left(\mu_{2}\right) \leq \psi\left(\mu_{1}\right)$ for all $\mu_{1}, \mu_{2} \in \mathbb{X}$, we can also get

$$
\psi\left(m \mu_{2}+\theta \eta\left(\mu_{1}, \mu_{2}, m\right)\right) \leq \psi\left(\mu_{1}\right) .
$$

Consequently,

$$
\psi\left(m \mu_{2}+\theta \eta\left(\mu_{1}, \mu_{2}, m\right)\right) \leq \max \left\{\psi\left(\mu_{1}\right), \psi\left(\mu_{2}\right)\right\} .
$$

That is, $\psi: \mathbb{X} \subseteq \mathbb{R}^{n} \rightarrow \mathbb{R}$ is generalized quasi $m$-preinvex function on $m$-invex set $\mathbb{X}$ with respect to $\eta$. 
Theorem 6. If $\psi_{i}: \mathbb{X} \subseteq \mathbb{R}^{n} \rightarrow \mathbb{R}(i=1,2, \ldots, n)$ are n-polynomial s-type m-preinvex functions on $\mathbb{X} w$.r.t $\eta: \mathbb{X} \times \mathbb{X} \times(0,1] \rightarrow \mathbb{R}$ for $s \in[0,1], m \in(0,1]$ and $\theta \in[0,1]$, then

$$
\psi=\max \left\{\psi_{i}, i=1,2, \ldots, n\right\}
$$

is also an n-polynomial s-type $m$-preinvex function on $\mathbb{X}$ w.r.t $\eta$ for $s \in[0,1]$ and $m \in(0,1]$.

Proof. The proof is obvious.

Theorem 7. If $\psi_{i}: \mathbb{X} \subseteq \mathbb{R}^{n} \rightarrow \mathbb{R}(i=1,2, \ldots, n)$ are $n$-polynomial s-type m-preinvex functions on $\mathbb{X}$ w.r.t $\eta: \mathbb{X} \times \mathbb{X} \times(0,1] \rightarrow \mathbb{R}$ for $s \in[0,1], m \in(0,1]$ and $\theta \in[0,1]$, then the function

$$
\psi=\sum_{i=1}^{n} \mu_{i} \psi_{i}, \mu_{i} \geq 0, \quad(i=1,2,3, \ldots, n)
$$

is also an n-polynomial s-type $m$-preinvex function on $\mathbb{X}$ w.r.t $\eta$ for $s \in[0,1]$ and $m \in(0,1]$.

Proof. The proof is obvious.

Theorem 8. Let $\psi: \mathbb{R}_{\circ} \rightarrow \mathbb{R}_{\circ}$ is an n-polynomial s-type m-preinvex function w.r.t $\eta: \mathbb{R}_{\circ} \times$ $\mathbb{R}_{\circ} \times(0,1] \rightarrow \mathbb{R}_{\circ}$ for $s \in[0,1], m \in(0,1]$ and $\theta \in[0,1]$. Assuming that $\psi$ is monotonic decreasing, $\eta$ is monotonic increasing regarding $m$ for fixed $\mu_{1}, \mu_{2} \in \mathbb{R}_{\circ}$ and $m_{1} \leq m_{2} \quad\left(m_{1}, m_{2} \in\right.$ $(0,1])$. If $\psi$ is an $n$-polynomial s-type $m_{1}$-preinvex function on $\mathbb{R}_{\circ}$ with respect to $\eta$, then $\psi$ is an $n$-polynomial s-type $m_{2}$-preinvex function on $\mathbb{R}_{\circ}$ with respect to $\eta$.

Proof. Since $\psi$ is an $n$-polynomial $s$-type $m_{1}$-preinvex function, for all $\mu_{1}, \mu_{2} \in \mathbb{R}_{\mathrm{o}}$,

$$
\psi\left(m_{1} \mu_{2}+\theta \eta\left(\mu_{1}, \mu_{2}, m_{1}\right)\right) \leq \frac{1}{n} \sum_{i=1}^{n}(1-(s(1-\theta)))^{i} \psi\left(\mu_{1}\right)+\frac{1}{n} \sum_{i=1}^{n} m_{1}^{i}\left(1-(s \theta)^{i}\right) \psi\left(\mu_{2}\right) .
$$

Combining the monotone decreasing of the function $\psi$ with the monotone increasing of the mapping $\eta$ regarding $m$ for fixed $\mu_{1}, \mu_{2} \in \mathbb{R}_{\circ}$ and $m_{1} \leq m_{2}$, it follows that

$$
\psi\left(m_{2} \mu_{2}+\theta \eta\left(\mu_{1}, \mu_{2}, m_{2}\right)\right) \leq \psi\left(m_{1} \mu_{2}+\theta \eta\left(\mu_{1}, \mu_{2}, m_{1}\right)\right)
$$

and

$$
\begin{aligned}
& \frac{1}{n} \sum_{i=1}^{n}(1-(s(1-\theta)))^{i} \psi\left(\mu_{1}\right)+\frac{1}{n} \sum_{i=1}^{n} m_{2}^{i}\left(1-(s \theta)^{i}\right) \psi\left(\mu_{2}\right) \\
& \leq \frac{1}{n} \sum_{i=1}^{n}(1-(s(1-\theta)))^{i} \psi\left(\mu_{1}\right)+\frac{1}{n} \sum_{i=1}^{n} m_{1}^{i}\left(1-(s \theta)^{i}\right) \psi\left(\mu_{2}\right) .
\end{aligned}
$$

Following the above inequalities, we have

$$
\psi\left(m_{2} \mu_{2}+\theta \eta\left(\mu_{1}, \mu_{2}, m_{2}\right)\right) \leq \frac{1}{n} \sum_{i=1}^{n}(1-(s(1-\theta)))^{i} \psi\left(\mu_{1}\right)+\frac{1}{n} \sum_{i=1}^{n} m_{2}^{i}\left(1-(s \theta)^{i}\right) \psi\left(\mu_{2}\right) .
$$

This is the required proof.

Theorem 9. Let $\psi, \varphi: \mathbb{X}=\left[\mu_{1}, \mu_{2}\right] \rightarrow \mathbb{R}$. If both functions are n-polynomial s-type m-preinvex, similarly ordered functions and $[1-(s(1-\theta))]+[1-s \theta] \leq 1$, for $s \in[0,1], m \in(0,1]$ and $\theta \in[0,1]$, the product of these functions is an n-polynomial s-type m-preinvex function. 
Proof. For $s \in[0,1], m \in(0,1]$ and $\theta \in[0,1]$, then

$$
\begin{aligned}
& \psi\left(m \mu_{2}+\theta \eta\left(\mu_{1}, \mu_{2}, m\right)\right) \varphi\left(m \mu_{2}+\theta \eta\left(\mu_{1}, \mu_{2}, m\right)\right) \\
& \leq\left[\frac{1}{n} \sum_{i=1}^{n}(1-(s(1-\theta)))^{i} \psi\left(\mu_{1}\right)+\frac{1}{n} \sum_{i=1}^{n} m^{i}\left(1-(s \theta)^{i}\right) \psi\left(\mu_{2}\right)\right] \\
& \times\left[\frac{1}{n} \sum_{i=1}^{n}(1-(s(1-\theta)))^{i} \varphi\left(\mu_{1}\right)+\frac{1}{n} \sum_{i=1}^{n} m^{i}\left(1-(s \theta)^{i}\right) \varphi\left(\mu_{2}\right)\right] \\
& \leq \frac{1}{n^{2}} \sum_{i=1}^{n}(1-(s(1-\theta)))^{2 i} \psi\left(\mu_{1}\right) \varphi\left(\mu_{1}\right)+\frac{1}{n^{2}} \sum_{i=1}^{n} m^{2 i}\left(1-(s \theta)^{i}\right)^{2} \psi\left(\mu_{2}\right) \varphi\left(\mu_{2}\right) \\
& +\frac{1}{n^{2}} \sum_{i=1}^{n} m^{i}\left(1-(s(1-\theta))^{i}\right)\left(1-(s \theta)^{i}\right)\left[\psi\left(\mu_{1}\right) \varphi\left(\mu_{2}\right)+\psi\left(\mu_{2}\right) \varphi\left(\mu_{1}\right)\right] \\
& \leq \frac{1}{n^{2}} \sum_{i=1}^{n}[1-(s(1-\theta))]^{2 i} \psi\left(\mu_{1}\right) \varphi\left(\mu_{1}\right)+\frac{1}{n^{2}} \sum_{i=1}^{n} m^{2 i}\left(1-(s \theta)^{i}\right)^{2} \psi\left(\mu_{2}\right) \varphi\left(\mu_{2}\right) \\
& +\frac{1}{n^{2}} \sum_{i=1}^{n} m^{i}(1-(s(1-\theta)))^{i}\left(1-(s \theta)^{i}\right)\left[\psi\left(\mu_{1}\right) \varphi\left(\mu_{1}\right)+\psi\left(\mu_{2}\right) \varphi\left(\mu_{2}\right)\right] \\
& =\left[\frac{1}{n} \sum_{i=1}^{n}(1-(s(1-\theta)))^{i} \psi\left(\mu_{1}\right) \varphi\left(\mu_{1}\right)+\frac{1}{n} \sum_{i=1}^{n} m^{i}\left(1-(s \theta)^{i}\right) \psi\left(\mu_{2}\right) \varphi\left(\mu_{2}\right)\right] \\
& \times\left(\frac{1}{n} \sum_{i=1}^{n}(1-(s(1-\theta)))^{i}+\frac{1}{n} \sum_{i=1}^{n} m^{i}\left(1-(s \theta)^{i}\right)\right) \\
& \leq \frac{1}{n} \sum_{i=1}^{n}(1-(s(1-\theta)))^{i} \psi\left(\mu_{1}\right) \varphi\left(\mu_{1}\right)+\frac{1}{n} \sum_{i=1}^{n} m^{i}\left(1-(s \theta)^{i}\right) \psi\left(\mu_{2}\right) \varphi\left(\mu_{2}\right) .
\end{aligned}
$$

Remark 3. Taking $n=m=1$ and $\eta\left(\mu_{1}, \mu_{2}, m\right)=\mu_{1}-m \mu_{2}$ in Theorem 5, then $\psi\left(\theta \mu_{1}+(1-\theta) \mu_{2}\right) \varphi\left(\theta \mu_{1}+(1-\theta) \mu_{2}\right) \leq[1-(s(1-\theta))] \psi\left(\mu_{1}\right) \varphi\left(\mu_{1}\right)+[1-s \theta] \psi\left(\mu_{2}\right) \varphi\left(\mu_{2}\right)$.

\section{Hermite-Hadamard Type Inequality via Generalized Preinvex Function}

In this section, we present a new version of the Hermite-Hadamard type inequality in the setting of $n$-polynomial $s$-type $m$-preinvex functions.

Theorem 10. Let $\mathbb{X}^{\circ} \subseteq \mathbb{R}$ be an open m-invex subset with respect to $\eta: \mathbb{X}^{\circ} \times \mathbb{X}^{\circ} \times(0,1] \rightarrow \mathbb{R}$ for some fixed $m \in(0,1]$ and $s \in[0,1]$, and let $\mu_{1}, \mu_{2} \in \mathbb{X}^{\circ}, \mu_{1}<\mu_{2}$ with $m \mu_{2}+\eta\left(\mu_{1}, \mu_{2}, m\right) \leq \mu_{2}$. Suppose $\psi:\left[m \mu_{2}+\eta\left(\mu_{1}, \mu_{2}, m\right), \mu_{2}\right]$ for all $m \in(0,1]$ and satisfies extended Condition-C, then

$$
\begin{aligned}
\psi\left(m \mu_{2}+\frac{1}{2} \eta\left(\mu_{1}, \mu_{2}, m\right)\right) \leq \frac{\frac{1}{n} \sum_{i=1}^{n}\left(1-\frac{s}{2}\right)^{i}}{\eta\left(\mu_{1}, \mu_{2}, m\right)}\left[\int_{m \mu_{2}}^{m \mu_{2}+\eta\left(\mu_{1}, \mu_{2}, m\right)} \psi(x) d x+m^{i} \int_{\frac{m \mu_{2}+\eta\left(\mu_{1}, \mu_{2}, m\right)}{m}}^{\mu_{2}} \psi(x) d x\right] \\
\leq \frac{1}{n} \sum_{i=1}^{n}(2-s)^{i}\left[\psi\left(\mu_{1}\right)+m^{i} \psi\left(\mu_{2}\right)\right] .
\end{aligned}
$$

Proof. Since $\mu_{1}, \mu_{2} \in \mathbb{X}^{\circ}$ and $\mathbb{X}^{\circ}$ is an $m$-invex set with respect to $\eta$, for every $m \in(0,1]$ and $\theta \in[0,1]$, we have $m \mu_{2}+\theta \eta\left(\mu_{1}, \mu_{2}, m\right) \in \mathbb{X}^{\circ}$. For the remaining, putting extended condition $C$ for $\eta, \theta=\frac{1}{2}$, using Definition 7 , and integrating over $[0,1]$, 


$$
\begin{aligned}
& \psi(m y+\theta \eta(x, y, m)) \leq \frac{1}{n} \sum_{i=1}^{n}(1-(s(1-\theta)))^{i} \psi(x)+\frac{1}{n} \sum_{i=1}^{n} m^{i}\left(1-(s \theta)^{i}\right) \psi(y) \\
& \psi\left(m y+\frac{1}{2} \eta(x, y, m)\right) \leq \frac{1}{n} \sum_{i=1}^{n}\left(1-\frac{s}{2}\right)^{i}\left[\psi(x)+m^{i} \psi(y)\right] .
\end{aligned}
$$

In the above inequality, we put $x=m \mu_{2}+\theta \eta\left(\mu_{1}, \mu_{2}, m\right)$ and $m y=m \mu_{2}+(1-$ $\theta) \eta\left(\mu_{1}, \mu_{2}, m\right)$, so first we prove the L.H.S. of the above inequality

$$
\psi\left(m y+\frac{1}{2} \eta(x, y, m)\right)=\psi\left(m \mu_{2}+\frac{1}{2} \eta\left(\mu_{1}, \mu_{2}, m\right)\right) .
$$

Thus, after inputting the value of $x$ and $y$, we obtain

$$
\begin{aligned}
& \psi\left(m y+\frac{1}{2} \eta(x, y, m)\right) \\
& =\psi\left(m \mu_{2}+(1-\theta) \eta\left(\mu_{1}, \mu_{2}, m\right)\right. \\
& \left.+\frac{1}{2} \eta\left(m \mu_{2}+\theta \eta\left(\mu_{1}, \mu_{2}, m\right), m \mu_{2}+(1-\theta) \eta\left(\mu_{1}, \mu_{2}, m\right), m\right)\right) .
\end{aligned}
$$

Now, by using extended Condition C, we have

$$
\begin{gathered}
\eta\left(m \mu_{2}+\theta \eta\left(\mu_{1}, \mu_{2}, m\right), m \mu_{2}+(1-\theta) \eta\left(\mu_{1}, \mu_{2}, m\right)\right)=(\theta-1+\theta) \eta\left(\mu_{1}, \mu_{2}, m\right) . \\
\eta\left(m \mu_{2}+\theta \eta\left(\mu_{1}, \mu_{2}, m\right), m \mu_{2}+(1-\theta) \eta\left(\mu_{1}, \mu_{2}, m\right)\right)=(2 \theta-1) \eta\left(\mu_{1}, \mu_{2}, m\right) .
\end{gathered}
$$

Now, we put the value of $\eta$ in (8), then as a result, we obtain

$$
\begin{gathered}
\psi\left(m y+\frac{1}{2} \eta(x, y, m)\right)=\psi\left(m \mu_{2}+(1-\theta) \eta\left(\mu_{1}, \mu_{2}, m\right)+\frac{1}{2}(2 \theta-1) \eta\left(\mu_{1}, \mu_{2}, m\right)\right) \\
\psi\left(m y+\frac{1}{2} \eta(x, y, m)\right)=\psi\left(m \mu_{2}+\left(1-\theta+\theta-\frac{1}{2}\right) \eta\left(\mu_{1}, \mu_{2}, m\right)\right) \\
\psi\left(m y+\frac{1}{2} \eta(x, y, m)\right)=\psi\left(m \mu_{2}+\frac{1}{2} \eta\left(\mu_{1}, \mu_{2}, m\right)\right) .
\end{gathered}
$$

Now,

$$
\begin{aligned}
& \psi\left(m \mu_{2}+\frac{1}{2} \eta\left(\mu_{1}, \mu_{2}, m\right)\right) \\
& \leq \frac{1}{n} \sum_{i=1}^{n}\left(1-\frac{s}{2}\right)^{i}\left[\int_{0}^{1} \psi\left(m \mu_{2}+\theta \eta\left(\mu_{1}, \mu_{2}, m\right)\right) d \theta+m^{i} \int_{0}^{1} \psi\left(\mu_{2}+\frac{(1-\theta)}{m} \eta\left(\mu_{1}, \mu_{2}, m\right)\right) d \theta\right] \\
& \leq \frac{1}{n} \sum_{i=1}^{n}\left(1-\frac{s}{2}\right)^{i} \frac{1}{\eta\left(\mu_{1}, \mu_{2}, m\right)}\left[\int_{m \mu_{2}}^{m \mu_{2}+\eta\left(\mu_{1}, \mu_{2}, m\right)} \psi(x) d x+m^{i} \int_{\frac{m \mu_{2}+\eta\left(\mu_{1}, \mu_{2}, m\right)}{m}}^{\mu_{2}} \psi(x) d x\right] .
\end{aligned}
$$

For the proof of the right side inequality, using the definition of $n$-polynomial s-type $m$-preinvexity, as a result we attain 


$$
\begin{aligned}
& \frac{1}{\eta\left(\mu_{1}, \mu_{2}, m\right)}\left[\int_{m \mu_{2}}^{m \mu_{2}+\eta\left(\mu_{1}, \mu_{2}, m\right)} \psi(x) d x+m^{i} \int_{\frac{m \mu_{2}+\eta\left(\mu_{1}, \mu_{2}, m\right)}{m}}^{\mu_{2}} \psi(x) d x\right] \\
& \leq\left[\int_{0}^{1} \psi\left(m \mu_{2}+\theta \eta\left(\mu_{1}, \mu_{2}, m\right)\right) d \theta+m^{i} \int_{0}^{1} \psi\left(\mu_{2}+\frac{(1-\theta)}{m} \eta\left(\mu_{1}, \mu_{2}, m\right)\right) d \theta\right] \\
& \leq \int_{0}^{1} \frac{1}{n} \sum_{i=1}^{n}(1-(s(1-\theta)))^{i} \psi\left(\mu_{1}\right) d \theta+\int_{0}^{1} \frac{1}{n} \sum_{i=1}^{n} m^{i}\left(1-(s \theta)^{i}\right) \psi\left(\mu_{2}\right) d \theta \\
& +\int_{0}^{1} \frac{1}{n} \sum_{i=1}^{n}\left(1-(s \theta)^{i}\right) \psi\left(\mu_{1}\right) d \theta+\int_{0}^{1} \frac{1}{n} \sum_{i=1}^{n} m^{i}(1-(s(1-\theta)))^{i} \psi\left(\mu_{2}\right) d \theta \\
& \left.\leq \frac{1}{n} \sum_{i=1}^{n}\left(\frac{2-s}{2}\right)^{i}\left[\psi\left(\mu_{1}\right)+\psi\left(\mu_{1}\right)+m^{i}\left(\psi\left(\mu_{2}\right)+\psi\left(\mu_{2}\right)\right)\right)\right] \\
& \leq \frac{1}{n} \sum_{i=1}^{n}(2-s)^{i}\left[\psi\left(\mu_{1}\right)+m^{i}\left(\psi\left(\mu_{2}\right)\right)\right] .
\end{aligned}
$$

This completes the proof.

Corollary 1. If $n=s=m=1$ and $\eta\left(\mu_{1}, \mu_{2}, m\right)=\mu_{1}-m \mu_{2}$ in Theorem 10, then we attain the simple Hermite-Hadamard inequality in [9].

\section{Refinements of Hermite-Hadamard Type Inequality}

First we derive two new lemmas with reference to differentiable preinvex functions. taking these two new equalities into consideration, several refinements of the HermiteHadamard type inequality are reviewed. The inequalities also considered some fundamental inequalities such as Hölder's inequality, Power-mean inequality, improved power-mean integral inequality and Hölder-Íscan inequality.

Lemma 3. Let $\psi:\left[\mu_{1}, m \mu_{1}+\eta\left(\frac{\mu_{2}}{c}, \mu_{1}, m\right)\right] \rightarrow \mathbb{R}$ be a differentiable mapping on $\left(\mu_{1}, m \mu_{1}+\right.$ $\left.\eta\left(\frac{\mu_{2}}{c}, \mu_{1}, m\right)\right)$ with $0<c \leq 1$ and $m \mu_{1}+\eta\left(\mu_{2}, \mu_{1}, m\right)>\mu_{1}>0$. If $\psi^{\prime} \in \mathcal{L}\left(\mu_{1}, m \mu_{1}+\right.$ $\left.\eta\left(\frac{\mu_{2}}{c}, \mu_{1}, m\right)\right)$ and for all $m \in(0,1]$, then the following equality holds in the preinvex setting:

$$
\begin{aligned}
& \frac{\psi\left(\mu_{1}\right)+\psi\left(m \mu_{1}+\eta\left(\frac{\mu_{2}}{c}, \mu_{1}, m\right)\right)}{2}-\frac{c}{\eta\left(\mu_{2}, c \mu_{1}, m\right)} \int_{\mu_{1}}^{m \mu_{1}+\eta\left(\frac{\mu_{2}}{c}, \mu_{1}, m\right)} \psi(x) d x \\
& =\frac{\eta\left(\mu_{2}, c \mu_{1}, m\right)}{2 c} \int_{0}^{1}(1-2 \theta) \psi^{\prime}\left(m \frac{\mu_{2}}{c}+\theta \eta\left(\mu_{1}, \frac{\mu_{2}}{c}, m\right)\right) d \theta .
\end{aligned}
$$

Proof. Let $\mu_{1}, \mu_{2} \in \mathbb{X}$. Since $\mathbb{X}$ is an $m$-invex set w.r.t $\eta, \forall \theta \in[0,1], \frac{\mu_{2}}{c}+\theta \eta\left(\mu_{1}, \frac{\mu_{2}}{c}\right) \in \mathbb{X}$. Integrating by parts,

$$
\begin{aligned}
& \frac{\eta\left(\mu_{2}, c \mu_{1}, m\right)}{2 c} \int_{0}^{1}(1-2 \theta) \psi^{\prime}\left(\frac{m \mu_{2}}{c}+\theta \eta\left(\mu_{1}, \frac{\mu_{2}}{c}, m\right)\right) d \theta \\
& =\frac{\eta\left(\mu_{2}, c \mu_{1}, m\right)}{2 c}\left[\left.\frac{(1-2 \theta) \psi\left(\frac{m \mu_{2}}{c}+\theta \eta\left(\mu_{1}, \frac{\mu_{2}}{c}\right)\right)}{\eta\left(\mu_{1}, \frac{\mu_{2}}{c}, m\right)}\right|_{0} ^{1}+2 \int_{0}^{1} \frac{\psi\left(\frac{m \mu_{2}}{c}+\theta \eta\left(\mu_{1}, \frac{\mu_{2}}{c}, m\right)\right)}{\eta\left(\mu_{1}, \frac{\mu_{2}}{c}, m\right)} d \theta\right] \\
& =\frac{\eta\left(\mu_{2}, c \mu_{1}, m\right)}{2 c}\left[\frac{c\left(\psi\left(\mu_{1}\right)+\psi\left(m \mu_{1}+\eta\left(\frac{\mu_{2}}{c}, \mu_{1}, m\right)\right)\right.}{\eta\left(\mu_{2}, c \mu_{1}\right)}\right. \\
& \left.-\frac{2 c}{\eta\left(\mu_{2}, c \mu_{1}\right)} \int_{0}^{1} \psi\left(\frac{m \mu_{2}}{c}+\theta \eta\left(\mu_{1}, \frac{\mu_{2}}{c}, m\right)\right) d \theta\right] \\
& =\frac{\psi\left(\mu_{1}\right)+\psi\left(m \mu_{1}+\eta\left(\frac{\mu_{2}}{c}, \mu_{1}, m\right)\right)}{2}-\frac{c}{\eta\left(\mu_{2}, c \mu_{1}, m\right)} \int_{\mu_{1}}^{m \mu_{1}+\eta\left(\frac{\mu_{2}}{c}, \mu_{1}, m\right)} \psi(x) d x .
\end{aligned}
$$

This completes the proof. 
Lemma 4. Let $\psi:\left[\mu_{1}, m \mu_{1}+\eta\left(\frac{\mu_{2}}{c}, \mu_{1}, m\right)\right] \rightarrow \mathbb{R}$ be a differentiable mapping on $\left(\mu_{1}, m \mu_{1}+\eta\right.$ $\left.\left(\frac{\mu_{2}}{c}, \mu_{1}, m\right)\right)$ with $0<c \leq 1$ and $m \mu_{1}+\eta\left(\mu_{2}, \mu_{1}, m\right)>\mu_{1}>0$. If $\psi^{\prime} \in \mathcal{L}\left(\mu_{1}, m \mu_{1}+\eta\left(\frac{\mu_{2}}{c}, \mu_{1}\right.\right.$, $m)$ ) and for all $m \in(0,1]$, then the following equality holds in the preinvex setting:

$$
\begin{aligned}
& \frac{c}{\eta\left(\mu_{2}, c \mu_{1}, m\right)} \int_{\mu_{1}}^{m \mu_{1}+\eta\left(\frac{\mu_{2}}{c}, \mu_{1}, m\right)} \psi(x) d x-\psi\left(\frac{2 m \mu_{1}+\eta\left(\mu_{2}, \mu_{1}, m\right)}{2 c}\right) \\
& =\frac{\eta\left(\mu_{2}, c \mu_{1}, m\right)}{c}\left\{\int_{0}^{1} \theta \psi^{\prime}\left(\frac{m \mu_{2}}{c}+\theta \eta\left(\mu_{1}, \frac{\mu_{2}}{c}, m\right)\right) d \theta-\int_{1 / 2}^{1} \psi^{\prime}\left(\frac{m \mu_{2}}{c}+\theta \eta\left(\mu_{1}, \frac{\mu_{2}}{c}, m\right)\right) d \theta\right\} .
\end{aligned}
$$

Proof. Let $\mu_{1}, \mu_{2} \in \mathbb{X}$. Since $\mathbb{X}$ is an $m$-invex set w.r.t $\eta, \forall \theta \in[0,1], \frac{\mu_{2}}{c}+\theta \eta\left(\mu_{1}, \frac{\mu_{2}}{c}\right) \in \mathbb{X}$. Using integrating by parts,

$$
\begin{aligned}
& \frac{\eta\left(\mu_{2}, c \mu_{1}, m\right)}{c}\left\{\int_{0}^{1} \theta \psi^{\prime}\left(\frac{m \mu_{2}}{c}+\theta \eta\left(\mu_{1}, \frac{\mu_{2}}{c}, m\right)\right) d \theta-\int_{1 / 2}^{1} \psi^{\prime}\left(\frac{m \mu_{2}}{c}+\theta \eta\left(\mu_{1}, \frac{\mu_{2}}{c}, m\right)\right) d \theta\right\} \\
& =\frac{\eta\left(\mu_{2}, c \mu_{1}, m\right)}{c} \times\left[\left.\frac{\theta \psi\left(\frac{m \mu_{2}}{c}+\theta \eta\left(\mu_{1}, \frac{\mu_{2}}{c}, m\right)\right)}{\eta\left(\mu_{1}, \frac{\mu_{2}}{c}, m\right)}\right|_{0} ^{1}-\int_{0}^{1} \frac{\psi\left(\frac{m \mu_{2}}{c}+\theta \eta\left(\mu_{1}, \frac{\mu_{2}}{c}, m\right)\right)}{\eta\left(\mu_{1}, \frac{\mu_{2}}{c}, m\right)} d \theta\right. \\
& \left.\quad-\left.\frac{\psi\left(\frac{m \mu_{2}}{c}+\theta \eta\left(\mu_{1}, \frac{\mu_{2}}{c}, m\right)\right)}{\eta\left(\mu_{1}, \frac{\mu_{2}}{c}, m\right)} d \theta\right|_{\frac{1}{2}} ^{1}\right] \\
& =\frac{\eta\left(\mu_{2}, c \mu_{1}, m\right)}{c}\left[\frac{c \psi\left(\mu_{1}\right)}{\eta\left(c \mu_{1}, \mu_{2}, m\right)}-\frac{c}{\eta\left(c \mu_{1}, \mu_{2}, m\right)} \int_{0}^{1} \psi\left(\frac{m \mu_{2}}{c}+\theta \eta\left(\mu_{1}, \frac{\mu_{2}}{c}, m\right)\right) d \theta\right. \\
& \left.-\frac{c}{\eta\left(c \mu_{1}, \mu_{2}, m\right)}\left(\psi\left(\mu_{1}\right)-\psi\left(\frac{\left(2 m \mu_{1}+\eta\left(\mu_{2}, \mu_{1}, m\right)\right)}{2 c}\right)\right)\right] \\
& =\frac{c}{\eta\left(\mu_{2}, c \mu_{1}, m\right)} \int_{\mu_{1}}^{m \mu_{1}+\eta\left(\frac{m \mu_{2}}{c}, \mu_{1}, m\right)} \psi(x) d x-\psi\left(\frac{2 m \mu_{1}+\eta\left(\mu_{2}, \mu_{1}, m\right)}{2 c}\right) .
\end{aligned}
$$

In this way, the proof is completed.

Theorem 11. Let $\psi$ be defined as in Theorem 10. If $\left|\psi^{\prime}\right|$ is an n-polynomial s-type m-preinvex function on $\mathbb{X}^{\circ}$ for some fixed $m \in(0,1]$ and $s \in[0,1]$, then for each $x \in\left(m \mu_{1}, m \mu_{1}+\eta\left(\mu_{2}, \mu_{1}, m\right)\right)$, the following inequality holds:

$$
\begin{aligned}
& \left|\frac{\psi\left(\mu_{1}\right)+\psi\left(m \mu_{1}+\eta\left(\frac{\mu_{2}}{c}, \mu_{1}, m\right)\right)}{2}-\frac{c}{\eta\left(\mu_{2}, c \mu_{1}, m\right)} \int_{\mu_{1}}^{m \mu_{1}+\eta\left(\frac{\mu_{2}}{c}, \mu_{1}, m\right)} \psi(x) d x\right| \\
& \leq \frac{\eta\left(\mu_{2}, c \mu_{1}, m\right)}{2 c}\left\{\frac{1}{n} \sum_{i=1}^{n}\left(\frac{2-s}{4}\right)^{i}\left[\left|\psi^{\prime}\left(\mu_{1}\right)\right|+m^{i}\left|\psi^{\prime}\left(\frac{\mu_{2}}{c}\right)\right|\right]\right\} .
\end{aligned}
$$

Proof. Using Lemma 3, one has

$$
\begin{aligned}
& \left|\frac{\psi\left(\mu_{1}\right)+\psi\left(m \mu_{1}+\eta\left(\frac{\mu_{2}}{c}, \mu_{1}, m\right)\right)}{2}-\frac{c}{\eta\left(\mu_{2}, c \mu_{1}, m\right)} \int_{\mu_{1}}^{m \mu_{1}+\eta\left(\frac{\mu_{2}}{c}, \mu_{1}, m\right)} \psi(x) d x\right| \\
& =\frac{\eta\left(\mu_{2}, c \mu_{1}, m\right)}{2 c} \int_{0}^{1}|1-2 \theta|\left|\psi^{\prime}\left(\frac{m \mu_{2}}{c}+\theta \eta\left(\mu_{1}, \frac{\mu_{2}}{c}, m\right)\right)\right| d \theta .
\end{aligned}
$$

Since $\left|\psi^{\prime}\right|$ is an $n$-polynomial s-type $m$-preinvex on $\left(\mu_{1}, \mu_{1}+\eta\left(\mu_{2}, \mu_{1}\right)\right)$, we have 


$$
\begin{aligned}
& \left|\frac{\psi\left(\mu_{1}\right)+\psi\left(m \mu_{1}+\eta\left(\frac{\mu_{2}}{c}, \mu_{1}, m\right)\right)}{2}-\frac{c}{\eta\left(\mu_{2}, c \mu_{1}, m\right)} \int_{\mu_{1}}^{m \mu_{1}+\eta\left(\frac{\mu_{2}}{c}, \mu_{1}, m\right)} \psi(x) d x\right| \\
& \leq \frac{\eta\left(\mu_{2}, c \mu_{1}, m\right)}{2 c} \int_{0}^{1}|1-2 \theta|\left[\frac{1}{n} \sum_{i=1}^{n}(1-s(1-\theta))^{i}\left|\psi^{\prime}\left(\mu_{1}\right)\right|+\frac{1}{n} \sum_{i=1}^{n} m^{i}\left(1-(s \theta)^{i}\right)\left|\psi^{\prime}\left(\frac{\mu_{2}}{c}\right)\right|\right] d \theta \\
& \leq \frac{\eta\left(\mu_{2}, c \mu_{1}, m\right)}{2 c}\left\{\left|\psi^{\prime}\left(\mu_{1}\right)\right| \int_{0}^{1}|1-2 \theta| \frac{1}{n} \sum_{i=1}^{n}(1-s(1-\theta))^{i} d \theta\right. \\
& \left.+\left|\psi^{\prime}\left(\frac{\mu_{2}}{c}\right)\right| \int_{0}^{1}|1-2 \theta| \frac{1}{n} \sum_{i=1}^{n} m^{i}\left(1-(s \theta)^{i}\right) d \theta\right\} .
\end{aligned}
$$

Since,

$$
\int_{0}^{1}(1-s(1-\theta))|1-2 \theta| d \theta=\int_{0}^{1}(1-s \theta)|1-2 \theta| d \theta=-\frac{s-2}{4} .
$$

Using these computations in (11), we get the desired result.

Corollary 2. Considering $n=s=1$ in Theorem 11, then the above theorem become

$$
\begin{aligned}
& \left|\frac{\psi\left(\mu_{1}\right)+\psi\left(m \mu_{1}+\eta\left(\frac{\mu_{2}}{c}, \mu_{1}, m\right)\right)}{2}-\frac{c}{\eta\left(\mu_{2}, c \mu_{1}, m\right)} \int_{\mu_{1}}^{m \mu_{1}+\eta\left(\frac{\mu_{2}}{c}, \mu_{1}, m\right)} \psi(x) d x\right| \\
& \leq \frac{\eta\left(\mu_{2}, c \mu_{1}, m\right)}{8 c}\left\{\left[\left|\psi^{\prime}\left(\mu_{1}\right)\right|+4 m\left|\psi^{\prime}\left(\frac{\mu_{2}}{c}\right)\right|\right]\right\} .
\end{aligned}
$$

Corollary 3. Considering $n=s=m=1$ in Theorem 11, then

$$
\begin{aligned}
& \left|\frac{\psi\left(\mu_{1}\right)+\psi\left(\mu_{1}+\eta\left(\frac{\mu_{2}}{c}, \mu_{1}\right)\right)}{2}-\frac{c}{\eta\left(\mu_{2}, c \mu_{1}\right)} \int_{\mu_{1}}^{\mu_{1}+\eta\left(\frac{\mu_{2}}{c}, \mu_{1}\right)} \psi(x) d x\right| \\
& \leq \frac{\eta\left(\mu_{2}, c \mu_{1}\right)}{8 c}\left\{\left[\left|\psi^{\prime}\left(\mu_{1}\right)\right|+4\left|\psi^{\prime}\left(\frac{\mu_{2}}{c}\right)\right|\right]\right\} .
\end{aligned}
$$

Corollary 4. considering $n=1$ and $\eta\left(\mu_{1}, \mu_{2}, m\right)=\mu_{1}-m \mu_{2}$ in Theorem 11, then

$$
\begin{aligned}
& \left|\frac{\psi\left(\mu_{1}\right)+\psi\left(\frac{\mu_{2}}{c}\right)}{2}-\frac{c}{\left(\mu_{2}-m c \mu_{1}\right)} \int_{\mu_{1}}^{\frac{\mu_{2}}{c}} \psi(x) d x\right| \\
\leq & \frac{\left(\mu_{2}-m c \mu_{1}\right)}{2 c}\left\{\frac{2-s}{4}\left[\left|\psi^{\prime}\left(\mu_{1}\right)\right|+m\left|\psi^{\prime}\left(\frac{\mu_{2}}{c}\right)\right|\right]\right\} .
\end{aligned}
$$

Corollary 5. Considering $n=s=1$ and $\eta\left(\mu_{1}, \mu_{2}, m\right)=\mu_{1}-m \mu_{2}$ in Theorem 11, then $\left|\frac{\psi\left(\mu_{1}\right)+\psi\left(\frac{\mu_{2}}{c}\right)}{2}-\frac{c}{\left(\mu_{2}-m c \mu_{1}\right)} \int_{\mu_{1}}^{\frac{\mu_{2}}{c}} \psi(x) d x\right| \leq \frac{\left(\mu_{2}-m c \mu_{1}\right)}{8 c}\left\{\left[\left|\psi^{\prime}\left(\mu_{1}\right)\right|+4 m\left|\psi^{\prime}\left(\frac{\mu_{2}}{c}\right)\right|\right]\right\}$.

Theorem 12. Let $\psi$ be defined as in Theorem 10. If $\left|\psi^{\prime}\right|$ is an n-polynomial s-type m-preinvex function on $\mathbb{X}^{\circ}$ for some fixed $m \in(0,1]$ and $s \in[0,1]$, then for each $x \in\left(m \mu_{1}, m \mu_{1}+\eta\left(\mu_{2}, \mu_{1}, m\right)\right)$. If $\left|\psi^{\prime}\right|^{q}$ is an n-polynomial s-type $m$-preinvex function on $\mathbb{X}^{\circ}$ for some fixed $m \in(0,1], s \in[0,1]$ and for $p, q>1, \frac{1}{q}+\frac{1}{p}=1$, then for each $x \in\left(m \mu_{1}, m \mu_{1}+\eta\left(\mu_{2}, \mu_{1}, m\right)\right)$ the following inequality holds: 


$$
\begin{aligned}
& \left|\frac{\psi\left(\mu_{1}\right)+\psi\left(m \mu_{1}+\eta\left(\frac{\mu_{2}}{c}, \mu_{1}, m\right)\right)}{2}-\frac{c}{\eta\left(\mu_{2}, c \mu_{1}, m\right)} \int_{\mu_{1}}^{\mu_{1}+\eta\left(\frac{\mu_{2}}{c}, \mu_{1}\right)} \psi(x) d x\right| \\
& \leq \frac{\eta\left(\mu_{2}, c \mu_{1}, m\right)}{2 c}\left[\frac{1}{p+1}\right]^{1 / p}\left\{\frac{1}{n} \sum_{i=1}^{n}\left(\frac{2-s}{2}\right)^{i}\left[\left|\psi^{\prime}\left(\mu_{1}\right)\right|^{q}+m^{i}\left|\psi^{\prime}\left(\frac{\mu_{2}}{c}\right)\right|^{q}\right]\right\}^{1 / q} .
\end{aligned}
$$

Proof. From Lemma 3 and using Hölder's inequality, we have

$$
\begin{aligned}
& \left|\frac{\psi\left(\mu_{1}\right)+\psi\left(m \mu_{1}+\eta\left(\frac{\mu_{2}}{c}, \mu_{1}, m\right)\right)}{2}-\frac{c}{\eta\left(\mu_{2}, c \mu_{1}, m\right)} \int_{\mu_{1}}^{m \mu_{1}+\eta\left(\frac{\mu_{2}}{c}, \mu_{1}, m\right)} \psi(x) d x\right| \\
& =\frac{\eta\left(\mu_{2}, c \mu_{1}, m\right)}{2 c} \int_{0}^{1}|1-2 \theta|\left|\psi^{\prime}\left(\frac{m \mu_{2}}{c}+\theta \eta\left(\mu_{1}, \frac{\mu_{2}}{c}, m\right)\right)\right| d \theta \\
& \leq \frac{\eta\left(\mu_{2}, c \mu_{1}, m\right)}{2 c}\left(\int_{0}^{1}|1-2 \theta|^{p} d \theta\right)^{1 / p}\left(\int_{0}^{1}\left|\psi^{\prime}\left(\frac{m \mu_{2}}{c}+\theta \eta\left(\mu_{1}, \frac{\mu_{2}}{c}, m\right)\right)\right|^{q} d \theta\right)^{1 / q} .
\end{aligned}
$$

Since $\left|\psi^{\prime}\right|^{q}$ is an $n$-polynomial s-type $m$-preinvex on $\left(\mu_{1}, m \mu_{1}+\eta\left(\mu_{2}, \mu_{1}, m\right)\right)$, we have

$$
\begin{aligned}
& \int_{0}^{1}\left|\psi^{\prime}\left(\frac{m \mu_{2}}{c}+\theta \eta\left(\mu_{1}, \frac{\mu_{2}}{c}, m\right)\right)\right|^{q} d \theta \\
& =\left|\psi^{\prime}\left(\mu_{1}\right)\right|^{q} \int_{0}^{1} \frac{1}{n} \sum_{i=1}^{n}(1-s(1-\theta))^{i} d \theta+\left|\psi^{\prime}\left(\frac{\mu_{2}}{c}\right)\right|^{q} \int_{0}^{1} \frac{1}{n} \sum_{i=1}^{n} m^{i}\left(1-(s \theta)^{i}\right) d \theta .
\end{aligned}
$$

Now, Equation (12) becomes

$$
\begin{aligned}
& \left|\frac{\psi\left(\mu_{1}\right)+\psi\left(m \mu_{1}+\eta\left(\frac{\mu_{2}}{c}, \mu_{1}, m\right)\right)}{2}-\frac{c}{\eta\left(\mu_{2}, c \mu_{1}, m\right)} \int_{\mu_{1}}^{m \mu_{1}+\eta\left(\frac{\mu_{2}}{c}, \mu_{1}, m\right)} \psi(x) d x\right| \\
& \leq \frac{\eta\left(\mu_{2}, c \mu_{1}, m\right)}{2 c}\left[\frac{1}{p+1}\right]^{1 / p} \\
& \times\left(\left|\psi^{\prime}\left(\mu_{1}\right)\right|^{q} \int_{0}^{1} \frac{1}{n} \sum_{i=1}^{n}(1-s(1-\theta))^{i} d \theta+\left|\psi^{\prime}\left(\frac{\mu_{2}}{c}\right)\right|^{q} \int_{0}^{1} \frac{1}{n} \sum_{i=1}^{n} m^{i}\left(1-(s \theta)^{i}\right) d \theta\right)^{1 / q} .
\end{aligned}
$$

Since,

$$
\begin{aligned}
& \int_{0}^{1}(1-s(1-\theta)) d \theta=\int_{0}^{1}(1-s \theta) d \theta=-\frac{s-2}{2} . \\
& \int_{0}^{1}|1-2 \theta|^{p} d \theta=\left[\frac{1}{p+1}\right] .
\end{aligned}
$$

Using the above computations in (13), we have the desired result.

Corollary 6. Considering $n=s=1$ in Theorem 12, then

$$
\begin{aligned}
& \left|\frac{\psi\left(\mu_{1}\right)+\psi\left(m \mu_{1}+\eta\left(\frac{\mu_{2}}{c}, \mu_{1}, m\right)\right)}{2}-\frac{c}{\eta\left(\mu_{2}, c \mu_{1}, m\right)} \int_{\mu_{1}}^{m \mu_{1}+\eta\left(\frac{\mu_{2}}{c}, \mu_{1}, m\right)} \psi(x) d x\right| \\
& \leq \frac{\eta\left(\mu_{2}, c \mu_{1}, m\right)}{2^{\frac{1+q}{q}} c}\left[\frac{1}{p+1}\right]^{1 / p}\left\{\left[\left|\psi^{\prime}\left(\mu_{1}\right)\right|^{q}+m\left|\psi^{\prime}\left(\frac{\mu_{2}}{c}\right)\right|^{q}\right]\right\}^{1 / q} .
\end{aligned}
$$


Corollary 7. Considering $n=s=m=1$ in Theorem 12, then

$$
\begin{aligned}
& \left|\frac{\psi\left(\mu_{1}\right)+\psi\left(\mu_{1}+\eta\left(\frac{\mu_{2}}{c}, \mu_{1}\right)\right)}{2}-\frac{c}{\eta\left(\mu_{2}, c \mu_{1}\right)} \int_{\mu_{1}}^{\mu_{1}+\eta\left(\frac{\mu_{2}}{c}, \mu_{1}\right)} \psi(x) d x\right| \\
& \leq \frac{\eta\left(\mu_{2}, c \mu_{1}\right)}{2^{\frac{1+q}{q}} c}\left[\frac{1}{p+1}\right]^{1 / p}\left\{\left[\left|\psi^{\prime}\left(\mu_{1}\right)\right|^{q}+\left|\psi^{\prime}\left(\frac{\mu_{2}}{c}\right)\right| q\right]\right\}^{1 / q} .
\end{aligned}
$$

Corollary 8. Considering $n=1$ and $\eta\left(\mu_{1}, \mu_{2}, m\right)=\mu_{1}-m \mu_{2}$ in Theorem 12, then

$$
\begin{aligned}
& \left|\frac{\psi\left(\mu_{1}\right)+\psi\left(\frac{\mu_{2}}{c}\right)}{2}-\frac{c}{\left(\mu_{2}-m c \mu_{1}\right)} \int_{\mu_{1}}^{\frac{\mu_{2}}{c}} \psi(x) d x\right| \\
& \leq \frac{\left(\mu_{2}-m c \mu_{1}\right)}{2 c}\left[\frac{1}{p+1}\right]^{1 / p}\left\{\frac{2-s}{2}\left[\left|\psi^{\prime}\left(\mu_{1}\right)\right|^{q}+m\left|\psi^{\prime}\left(\frac{\mu_{2}}{c}\right)\right|^{q}\right]\right\}^{1 / q} .
\end{aligned}
$$

Corollary 9. Putting $n=s=1$ and $\eta\left(\mu_{1}, \mu_{2}\right)=\mu_{1}-\mu_{2}$ in Theorem 12, then

$$
\begin{aligned}
& \left|\frac{\psi\left(\mu_{1}\right)+\psi\left(\frac{\mu_{2}}{c}\right)}{2}-\frac{c}{\left(\mu_{2}-m c \mu_{1}\right)} \int_{\mu_{1}}^{\frac{\mu_{2}}{c}} \psi(x) d x\right| \\
& \leq \frac{\left(\mu_{2}-m c \mu_{1}\right)}{2^{\frac{1+q}{q}} c}\left[\frac{1}{p+1}\right]^{1 / p}\left\{\left[\left|\psi^{\prime}\left(\mu_{1}\right)\right|^{q}+m\left|\psi^{\prime}\left(\frac{\mu_{2}}{c}\right)\right|^{q}\right]\right\}^{1 / q} .
\end{aligned}
$$

Theorem 13. Let $\psi$ be defined as in Theorem 10. If $\left|\psi^{\prime}\right|$ is an n-polynomial s-type m-preinvex function on $\mathbb{X}^{\circ}$ for some fixed $m \in(0,1]$ and $s \in[0,1]$, then for each $x \in\left(m \mu_{1}, m \mu_{1}+\eta\left(\mu_{2}, \mu_{1}, m\right)\right)$. If $\left|\psi^{\prime}\right|^{q}$ is an $n$-polynomial s-type m-preinvex function on $\mathbb{X}^{\circ}$ for some fixed $m \in(0,1], s \in[0,1]$ and for $p, q>1, \frac{1}{q}+\frac{1}{p}=1$, then for each $x \in\left(m \mu_{1}, m \mu_{1}+\eta\left(\mu_{2}, \mu_{1}, m\right)\right)$, the following inequality holds:

$$
\begin{aligned}
& \left|\frac{\psi\left(\mu_{1}\right)+\psi\left(m \mu_{1}+\eta\left(\frac{\mu_{2}}{c}, \mu_{1}, m\right)\right)}{2}-\frac{c}{\eta\left(\mu_{2}, c \mu_{1}, m\right)} \int_{\mu_{1}}^{m \mu_{1}+\eta\left(\frac{\mu_{2}}{c}, \mu_{1}, m\right)} \psi(x) d x\right| \\
& \leq \frac{\eta\left(\mu_{2}, c \mu_{1}, m\right)}{c}\left[\frac{1}{2\left(\frac{p+1}{p}\right)}\right]\left\{\frac{1}{n} \sum_{i=1}^{n}\left(\frac{2-s}{4}\right)^{i}\left[\left|\psi^{\prime}\left(\mu_{1}\right)\right|^{q}+m^{i}\left|\psi^{\prime}\left(\frac{\mu_{2}}{c}\right)\right|^{q}\right]\right\}^{1 / q} .
\end{aligned}
$$

Proof. Using Lemma 3 and Hölder's inequality, one has

$$
\begin{aligned}
& \left|\frac{\psi\left(\mu_{1}\right)+\psi\left(m \mu_{1}+\eta\left(\frac{\mu_{2}}{c}, \mu_{1}, m\right)\right)}{2}-\frac{c}{\eta\left(\mu_{2}, c \mu_{1}, m\right)} \int_{\mu_{1}}^{\mu_{1}+\eta\left(\frac{\mu_{2}}{c}, \mu_{1}\right)} \psi(x) d x\right| \\
& \leq \frac{\eta\left(\mu_{2}, c \mu_{1}, m\right)}{2 c} \int_{0}^{1}|1-2 \theta|\left|\psi^{\prime}\left(\frac{m \mu_{2}}{c}+\theta \eta\left(\mu_{1}, \frac{\mu_{2}}{c}, m\right)\right)\right| d \theta \\
& =\frac{\eta\left(\mu_{2}, c \mu_{1}, m\right)}{2 c} \int_{0}^{1}|1-2 \theta|^{1 / p}|1-2 \theta|^{1 / q}\left|\psi^{\prime}\left(\frac{m \mu_{2}}{c}+\theta \eta\left(\mu_{1}, \frac{\mu_{2}}{c}, m\right)\right)\right| d \theta \\
& \leq \frac{\eta\left(\mu_{2}, c \mu_{1}, m\right)}{2 c}\left(\int_{0}^{1}|1-2 \theta| d \theta\right)^{1 / p}\left(\left.\int_{0}^{1}|1-2 \theta|\left|\psi^{\prime}\left(\frac{m \mu_{2}}{c}+\theta \eta\left(\mu_{1}, \frac{\mu_{2}}{c}, m\right)\right)\right|\right|^{q} d \theta\right)^{1 / q} .
\end{aligned}
$$

Since $\left|\psi^{\prime}\right|^{q}$ is an $n$-polynomial $s$-type $m$-preinvex on $\left(\mu_{1}, m \mu_{1}+\eta\left(\mu_{2}, \mu_{1}, m\right)\right)$, we have

$$
\begin{aligned}
& \int_{0}^{1}\left|\psi^{\prime}\left(\frac{m \mu_{2}}{c}+\theta \eta\left(\mu_{1}, \frac{\mu_{2}}{c}, m\right)\right)\right|^{q} d \theta \\
& \quad=\left|\psi^{\prime}\left(\mu_{1}\right)\right|^{q} \int_{0}^{1} \frac{1}{n} \sum_{i=1}^{n}(1-s(1-\theta))^{i} d \theta+\left|\psi^{\prime}\left(\frac{\mu_{2}}{c}\right)\right|^{q} \int_{0}^{1} \frac{1}{n} \sum_{i=1}^{n} m^{i}\left(1-(s \theta)^{i}\right) d \theta .
\end{aligned}
$$


Now, Equation (15) becomes

$$
\begin{aligned}
& \left|\frac{\psi\left(\mu_{1}\right)+\psi\left(m \mu_{1}+\eta\left(\frac{\mu_{2}}{c}, \mu_{1}, m\right)\right)}{2}-\frac{c}{\eta\left(\mu_{2}, c \mu_{1}, m\right)} \int_{\mu_{1}}^{m \mu_{1}+\eta\left(\frac{\mu_{2}}{c}, \mu_{1}, m\right)} \psi(x) d x\right| \\
& \leq \frac{\eta\left(\mu_{2}, c \mu_{1}, m\right)}{2 c}\left(\int_{0}^{1}|1-2 \theta| d \theta\right)^{1 / p}\left(\left|\psi^{\prime}\left(\mu_{1}\right)\right|^{q} \int_{0}^{1}|1-2 \theta| \frac{1}{n} \sum_{i=1}^{n}(1-s(1-\theta))^{i} d \theta\right. \\
& \left.+\left|\psi^{\prime}\left(\frac{\mu_{2}}{c}\right)\right|^{q} \int_{0}^{1}|1-2 \theta| \frac{1}{n} \sum_{i=1}^{n} m^{i}\left(1-(s k)^{i}\right) d \theta\right)^{1 / q} .
\end{aligned}
$$

Since,

$$
\begin{aligned}
& \int_{0}^{1}|1-2 \theta|(1-s(1-\theta)) d \theta=\int_{0}^{1}|1-2 \theta|(1-s \theta) d \theta=-\frac{s-2}{4} . \\
& \int_{0}^{1}|1-2 \theta| d \theta=\frac{1}{2} .
\end{aligned}
$$

The proof of the Theorem gets completed by using these computations in (17).

Corollary 10. Putting $n=s=1$ in Theorem 13 , then

$$
\begin{aligned}
& \left|\frac{\psi\left(\mu_{1}\right)+\psi\left(m \mu_{1}+\eta\left(\frac{\mu_{2}}{c}, \mu_{1}, m\right)\right)}{2}-\frac{c}{\eta\left(\mu_{2}, c \mu_{1}, m\right)} \int_{\mu_{1}}^{m \mu_{1}+\eta\left(\frac{\mu_{2}}{c}, \mu_{1}, m\right)} \psi(x) d x\right| \\
& \leq \frac{\eta\left(\mu_{2}, c \mu_{1}\right)}{c}\left[\frac{1}{2\left(\frac{2 q+1}{q}\right)}\right]\left\{\left[\left|\psi^{\prime}\left(\mu_{1}\right)\right|^{q}+m\left|\psi^{\prime}\left(\frac{\mu_{2}}{c}\right)\right| q\right]\right\}^{1 / q} .
\end{aligned}
$$

Corollary 11. Choosing $n=s=m=1$ in Theorem 13, then

$$
\begin{aligned}
& \left|\frac{\psi\left(\mu_{1}\right)+\psi\left(\mu_{1}+\eta\left(\frac{\mu_{2}}{c}, \mu_{1}\right)\right)}{2}-\frac{c}{\eta\left(\mu_{2}, c \mu_{1}\right)} \int_{\mu_{1}}^{\mu_{1}+\eta\left(\frac{\mu_{2}}{c}, \mu_{1}\right)} \psi(x) d x\right| \\
& \leq \frac{\eta\left(\mu_{2}, c \mu_{1}\right)}{c}\left[\frac{1}{2\left(\frac{2 q+1}{q}\right)}\right]\left\{\left[\left|\psi^{\prime}\left(\mu_{1}\right)\right|^{q}+\left|\psi^{\prime}\left(\frac{\mu_{2}}{c}\right)\right|^{q}\right]\right\}^{1 / q} .
\end{aligned}
$$

Corollary 12. Considering $n=1$ and $\eta\left(\mu_{1}, \mu_{2}, m\right)=\mu_{1}-m \mu_{2}$ in Theorem 13, then

$$
\begin{aligned}
& \left|\frac{\psi\left(\mu_{1}\right)+\psi\left(\frac{\mu_{2}}{c}\right)}{2}-\frac{c}{\left(\mu_{2}-m c \mu_{1}\right)} \int_{\mu_{1}}^{\frac{\mu_{2}}{c}} \psi(x) d x\right| \\
& \leq \frac{\left(\mu_{2}-m c \mu_{1}\right)}{c}\left[\frac{1}{2\left(\frac{p+1}{p}\right)}\right]\left\{\frac{2-s}{4}\left[\left|\psi^{\prime}\left(\mu_{1}\right)\right|^{q}+m\left|\psi^{\prime}\left(\frac{\mu_{2}}{c}\right)\right|^{q}\right]\right\}^{1 / q} .
\end{aligned}
$$

Corollary 13. Putting $n=s=1$ and $\eta\left(\mu_{1}, \mu_{2}, m\right)=\mu_{1}-m \mu_{2}$ in Theorem 13, then

$$
\begin{aligned}
& \left|\frac{\psi\left(\mu_{1}\right)+\psi\left(\frac{\mu_{2}}{c}\right)}{2}-\frac{c}{\left(\mu_{2}-m c \mu_{1}\right)} \int_{\mu_{1}}^{\frac{\mu_{2}}{c}} \psi(x) d x\right| \\
& \leq \frac{\left(\mu_{2}-m c \mu_{1}\right)}{c}\left[\frac{1}{2\left(\frac{2 q+1}{q}\right)}\right]\left\{\left[\left|\psi^{\prime}\left(\mu_{1}\right)\right|^{q}+m\left|\psi^{\prime}\left(\frac{\mu_{2}}{c}\right)\right|^{q}\right]\right\}^{1 / q} .
\end{aligned}
$$

Theorem 14. Let $\psi$ be defined as in Theorem 10. If $\left|\psi^{\prime}\right|$ is an n-polynomial s-type m-preinvex function on $\mathbb{X}^{\circ}$ for some fixed $m \in(0,1]$ and $s \in[0,1]$, then for each $x \in\left(m \mu_{1}, m \mu_{1}+\eta\left(\mu_{2}, \mu_{1}, m\right)\right)$. If $\left|\psi^{\prime}\right|^{q}$ is an n-polynomial s-type m-preinvex function on $\mathbb{X}^{\circ}$ for some fixed $m \in(0,1], s \in[0,1]$ 
and for $p, q>1, \frac{1}{q}+\frac{1}{p}=1$, then for each $x \in\left(m \mu_{1}, m \mu_{1}+\eta\left(\mu_{2}, \mu_{1}, m\right)\right)$, the following inequality holds:

$$
\begin{aligned}
& \left|\frac{\psi\left(\mu_{1}\right)+\psi\left(m \mu_{1}+\eta\left(\frac{\mu_{2}}{c}, \mu_{1}, m\right)\right)}{2}-\frac{c}{\eta\left(\mu_{2}, c \mu_{1}, m\right)} \int_{\mu_{1}}^{m \mu_{1}+\eta\left(\frac{\mu_{2}}{c}, \mu_{1}, m\right)} \psi(x) d x\right| \\
& \leq \frac{\eta\left(\mu_{2}, c \mu_{1}, m\right)}{2 c}\left[\frac{1}{2(p+1)}\right]^{1 / p}\left[\left\{\sum_{i=1}^{n}\left(\frac{3-2 s}{6}\right)^{i}\left|\psi^{\prime}\left(\mu_{1}\right)\right|^{q}+\sum_{i=1}^{n}\left(\frac{3-s}{6}\right)^{i} m^{i}\left|\psi^{\prime}\left(\frac{\mu_{2}}{c}\right)\right|^{q}\right\}^{1 / q}\right. \\
& \left.+\left\{\frac{1}{n} \sum_{i=1}^{n}\left(\frac{3-s}{6}\right)^{i}\left|\psi^{\prime}\left(\mu_{1}\right)\right|^{q}+\frac{1}{n} \sum_{i=1}^{n}\left(\frac{3-2 s}{6}\right)^{i} m^{i}\left|\psi^{\prime}\left(\frac{\mu_{2}}{c}\right)\right|^{q}\right\}^{1 / q}\right] .
\end{aligned}
$$

Proof. From Lemma 3 and using Hölder-Íscan inequality, we have

$$
\begin{aligned}
& \left|\frac{\psi\left(\mu_{1}\right)+\psi\left(m \mu_{1}+\eta\left(\frac{\mu_{2}}{c}, \mu_{1}, m\right)\right)}{2}-\frac{c}{\eta\left(\mu_{2}, c \mu_{1}, m\right)} \int_{\mu_{1}}^{m \mu_{1}+\eta\left(\frac{\mu_{2}}{c}, \mu_{1}, m\right)} \psi(x) d x\right| \\
& \leq \frac{\eta\left(\mu_{2}, c \mu_{1}, m\right)}{2 c}\left[\left(\int_{0}^{1}(1-\theta)|1-2 \theta|^{p} d \theta\right)^{1 / p}\left(\int_{0}^{1}(1-\theta)\left|\psi^{\prime}\left(\frac{m \mu_{2}}{c}+\theta \eta\left(\mu_{1}, \frac{\mu_{2}}{c}, m\right)\right)\right|^{q} d \theta\right)^{1 / q}\right. \\
& \left.+\left(\int_{0}^{1} \theta|1-2 \theta|^{p} d \theta\right)^{1 / p}\left(\left.\int_{0}^{1} \theta\left|\psi^{\prime}\left(\frac{m \mu_{2}}{c}+\theta \eta\left(\mu_{1}, \frac{\mu_{2}}{c}, m\right)\right)\right|\right|^{q} d \theta\right)^{1 / q}\right]
\end{aligned}
$$

Since $\left|\psi^{\prime}\right|^{q}$ is an $n$-polynomial s-type $m$-preinvex on $\left(\mu_{1}, m \mu_{1}+\eta\left(\mu_{2}, \mu_{1}, m\right)\right)$, we have

$$
\begin{aligned}
& \int_{0}^{1}\left|\psi^{\prime}\left(\frac{m \mu_{2}}{c}+\theta \eta\left(\mu_{1}, \frac{\mu_{2}}{c}, m\right)\right)\right|^{q} d \theta \\
& =\left|\psi^{\prime}\left(\mu_{1}\right)\right|^{q} \int_{0}^{1} \frac{1}{n} \sum_{i=1}^{n}(1-s(1-\theta))^{i} d \theta+\left|\psi^{\prime}\left(\frac{\mu_{2}}{c}\right)\right|^{q} \int_{0}^{1} \frac{1}{n} \sum_{i=1}^{n} m^{i}\left(1-(s \theta)^{i}\right) d \theta
\end{aligned}
$$

Now, Equation (18) becomes

$$
\begin{aligned}
& \left|\frac{\psi\left(\mu_{1}\right)+\psi\left(m \mu_{1}+\eta\left(\frac{\mu_{2}}{c}, \mu_{1}, m\right)\right)}{2}-\frac{c}{\eta\left(\mu_{2}, c \mu_{1}, m\right)} \int_{\mu_{1}}^{m \mu_{1}+\eta\left(\frac{\mu_{2}}{c}, \mu_{1}, m\right)} \psi(x) d x\right| \\
& \leq \frac{\eta\left(\mu_{2}, c \mu_{1}, m\right)}{2 c}\left[( \int _ { 0 } ^ { 1 } ( 1 - \theta ) | 1 - 2 \theta | ^ { p } d \theta ) ^ { 1 / p } \left(\left|\psi^{\prime}\left(\mu_{1}\right)\right|^{q} \int_{0}^{1}(1-\theta) \frac{1}{n} \sum_{i=1}^{n}(1-s(1-\theta))^{i} d \theta\right.\right. \\
& \left.+\left|\psi^{\prime}\left(\frac{\mu_{2}}{c}\right)\right|^{q} \int_{0}^{1}(1-\theta) \frac{1}{n} \sum_{i=1}^{n} m^{i}\left(1-(s \theta)^{i}\right) d \theta\right)^{1 / q}+\left(\int_{0}^{1} \theta|1-2 \theta|^{p} d \theta\right)^{1 / p} \\
& \left.\times\left(\left|\psi^{\prime}\left(\mu_{1}\right)\right|^{q} \int_{0}^{1} \theta \frac{1}{n} \sum_{i=1}^{n}(1-s(1-\theta))^{i} d \theta+\left|\psi^{\prime}\left(\frac{\mu_{2}}{c}\right)\right|^{q} \int_{0}^{1} \theta \frac{1}{n} \sum_{i=1}^{n} m^{i}\left(1-(s \theta)^{i}\right) d \theta\right)^{1 / q}\right] .
\end{aligned}
$$

Since,

$$
\begin{gathered}
\int_{0}^{1}(1-\theta)(1-s(1-\theta)) d \theta=\int_{0}^{1} \theta(1-s \theta) d \theta=-\frac{2 s-3}{6} . \\
\int_{0}^{1} \theta(1-s(1-\theta)) d \theta=\int_{0}^{1}(1-\theta)(1-s \theta) d \theta=-\frac{s-3}{6} . \\
\int_{0}^{1} \theta|1-2 \theta|^{p} d \theta=\int_{0}^{1}(1-\theta)|1-2 \theta|^{p} d \theta=\left[\frac{1}{2(p+1)}\right] .
\end{gathered}
$$

Consequently, using the above computations in (20) lead us to the desired result. 
Corollary 14. Considering $n=s=1$ in Theorem 14 , then

$$
\begin{aligned}
& \left|\frac{\psi\left(\mu_{1}\right)+\psi\left(m \mu_{1}+\eta\left(\frac{\mu_{2}}{c}, \mu_{1}, m\right)\right)}{2}-\frac{c}{\eta\left(\mu_{2}, c \mu_{1}, m\right)} \int_{\mu_{1}}^{m \mu_{1}+\eta\left(\frac{\mu_{2}}{c}, \mu_{1}, m\right)} \psi(x) d x\right| \\
& \leq \frac{\eta\left(\mu_{2}, c \mu_{1}, m\right)}{2 c}\left[\frac{1}{2(p+1)}\right]^{1 / p}\left[\left\{\frac{1}{6}\left|\psi^{\prime}\left(\mu_{1}\right)\right|^{q}+\frac{1}{3} m\left|\psi^{\prime}\left(\frac{\mu_{2}}{c}\right)\right|^{q}\right\}^{1 / q}\right. \\
& \left.+\left\{\frac{1}{3}\left|\psi^{\prime}\left(\mu_{1}\right)\right|^{q}+\frac{1}{6} m\left|\psi^{\prime}\left(\frac{\mu_{2}}{c}\right)\right|^{q}\right\}^{1 / q}\right] .
\end{aligned}
$$

Corollary 15. Considering $n=s=m=1$ in Theorem 14 , then

$$
\begin{aligned}
& \left|\frac{\psi\left(\mu_{1}\right)+\psi\left(\mu_{1}+\eta\left(\frac{\mu_{2}}{c}, \mu_{1}\right)\right)}{2}-\frac{c}{\eta\left(\mu_{2}, c \mu_{1}\right)} \int_{\mu_{1}}^{\mu_{1}+\eta\left(\frac{\mu_{2}}{c}, \mu_{1}\right)} \psi(x) d x\right| \\
& \leq \frac{\eta\left(\mu_{2}, c \mu_{1}\right)}{2 c}\left[\frac{1}{2(p+1)}\right]^{1 / p}\left[\left\{\frac{1}{6}\left|\psi^{\prime}\left(\mu_{1}\right)\right|^{q}+\frac{1}{3}\left|\psi^{\prime}\left(\frac{\mu_{2}}{c}\right)\right|^{q}\right\}^{1 / q}\right. \\
& \left.+\left\{\frac{1}{3}\left|\psi^{\prime}\left(\mu_{1}\right)\right|^{q}+\frac{1}{6}\left|\psi^{\prime}\left(\frac{\mu_{2}}{c}\right)\right|^{q}\right\}^{1 / q}\right] .
\end{aligned}
$$

Corollary 16. Considering $n=1$ and $\eta\left(\mu_{1}, \mu_{2}, m\right)=\mu_{1}-m \mu_{2}$ in Theorem 14 , then

$$
\begin{aligned}
& \left|\frac{\psi\left(\mu_{1}\right)+\psi\left(\frac{\mu_{2}}{c}\right)}{2}-\frac{c}{\left(\mu_{2}-m c \mu_{1}\right)} \int_{\mu_{1}}^{\frac{\mu_{2}}{c}} \psi(x) d x\right| \\
& \leq \frac{\left(\mu_{2}-m c \mu_{1}\right)}{2 c}\left[\frac{1}{2(p+1)}\right]^{1 / p}\left[\left\{\frac{3-2 s}{6}\left|\psi^{\prime}\left(\mu_{1}\right)\right|^{q}+\frac{3-s}{6} m\left|\psi^{\prime}\left(\frac{\mu_{2}}{c}\right)\right|^{q}\right\}^{1 / q}\right. \\
& \left.+\left\{\frac{3-s}{6}\left|\psi^{\prime}\left(\mu_{1}\right)\right|^{q}+\frac{3-2 s}{6} m\left|\psi^{\prime}\left(\frac{\mu_{2}}{c}\right)\right|^{q}\right\}^{1 / q}\right] .
\end{aligned}
$$

Corollary 17. Considering $n=s=1$ and $\eta\left(\mu_{1}, \mu_{2}, m\right)=\mu_{1}-m \mu_{2}$ in Theorem 14, then

$$
\begin{aligned}
& \left|\frac{\psi\left(\mu_{1}\right)+\psi\left(\frac{\mu_{2}}{c}\right)}{2}-\frac{c}{\left(\mu_{2}-m c \mu_{1}\right)} \int_{\mu_{1}}^{\frac{\mu_{2}}{c}} \psi(x) d x\right| \\
& \leq \frac{\left(\mu_{2}-m c \mu_{1}\right)}{2 c}\left[\frac{1}{2(p+1)}\right]^{1 / p}\left[\left\{\frac{1}{6}\left|\psi^{\prime}\left(\mu_{1}\right)\right|^{q}+\frac{1}{3} m\left|\psi^{\prime}\left(\frac{\mu_{2}}{c}\right)\right|^{q}\right\}^{1 / q}\right. \\
& \left.+\left\{\frac{1}{3}\left|\psi^{\prime}\left(\mu_{1}\right)\right|^{q}+\frac{1}{6} m\left|\psi^{\prime}\left(\frac{\mu_{2}}{c}\right)\right|^{q}\right\}^{1 / q}\right] .
\end{aligned}
$$

Theorem 15. Let $\psi$ be defined as in Theorem 10. If $\left|\psi^{\prime}\right|$ is an n-polynomial s-type m-preinvex function on $\mathbb{X}^{\circ}$ for some fixed $m \in(0,1]$ and $s \in[0,1]$, then for each $x \in\left(m \mu_{1}, m \mu_{1}+\eta\left(\mu_{2}, \mu_{1}, m\right)\right)$. If $\left|\psi^{\prime}\right|^{q}$ is an $n$-polynomial s-type $m$-preinvex function on $\mathbb{X}^{\circ}$ for some fixed $m \in(0,1], s \in[0,1]$ and for $q \geq 1$, then for each $x \in\left(m \mu_{1}, m \mu_{1}+\eta\left(\mu_{2}, \mu_{1}, m\right)\right)$, the following inequality holds: 


$$
\begin{aligned}
& \left|\frac{\psi\left(\mu_{1}\right)+\psi\left(m \mu_{1}+\eta\left(\frac{\mu_{2}}{c}, \mu_{1}, m\right)\right)}{2}-\frac{c}{\eta\left(\mu_{2}, c \mu_{1}, m\right)} \int_{\mu_{1}}^{m \mu_{1}+\eta\left(\frac{\mu_{2}}{c}, \mu_{1}, m\right)} \psi(x) d x\right| \\
& \leq \frac{\eta\left(\mu_{2}, c \mu_{1}, m\right)}{2 c}\left[\frac{1}{4}\right]^{1-1 / q}\left[\left\{\frac{1}{n} \sum_{i=1}^{n}\left(\frac{4-3 s}{16}\right)^{i}\left|\psi^{\prime}\left(\mu_{1}\right)\right|^{q}+\frac{1}{n} \sum_{i=1}^{n}\left(\frac{4-s}{16}\right)^{i} m^{i}\left|\psi^{\prime}\left(\frac{\mu_{2}}{c}\right)\right|^{q}\right\}^{1 / q}\right. \\
& \left.+\left\{\frac{1}{n} \sum_{i=1}^{n}\left(\frac{4-s}{16}\right)^{i}\left|\psi^{\prime}\left(\mu_{1}\right)\right|^{q}+\frac{1}{n} \sum_{i=1}^{n}\left(\frac{4-3 s}{16}\right)^{i} m^{i}\left|\psi^{\prime}\left(\frac{\mu_{2}}{c}\right)\right|^{q}\right\}^{1 / q}\right] .
\end{aligned}
$$

Proof. From Lemma 3 and using improved power-mean inequality, we have

$$
\begin{aligned}
& \left|\frac{\psi\left(\mu_{1}\right)+\psi\left(m \mu_{1}+\eta\left(\frac{\mu_{2}}{c}, \mu_{1}, m\right)\right)}{2}-\frac{c}{\eta\left(\mu_{2}, c \mu_{1}, m\right)} \int_{\mu_{1}}^{m \mu_{1}+\eta\left(\frac{\mu_{2}}{c}, \mu_{1}, m\right)} \psi(x) d x\right| \\
& \leq \frac{\eta\left(\mu_{2}, c \mu_{1}, m\right)}{2 c}\left[\left(\int_{0}^{1}(1-\theta)|1-2 \theta| d \theta\right)^{1-1 / q}\right. \\
& \times\left(\int_{0}^{1}(1-\theta)\left|1-2 \theta \| \psi^{\prime}\left(\frac{m \mu_{2}}{c}+\theta \eta\left(\mu_{1}, \frac{\mu_{2}}{c}, m\right)\right)\right|^{q} d \theta\right)^{1 / q} \\
& \left.+\left(\int_{0}^{1} \theta|1-2 \theta| d \theta\right)^{1-1 / q}\left(\int_{0}^{1} \theta|1-2 \theta|\left|\psi^{\prime}\left(\frac{m \mu_{2}}{c}+\theta \eta\left(\mu_{1}, \frac{\mu_{2}}{c}, m\right)\right)\right|^{q} d \theta\right)^{1 / q}\right] .
\end{aligned}
$$

Since $\left|\psi^{\prime}\right|^{q}$ is an $n$-polynomial s-type $m$-preinvex on $\left(\mu_{1}, m \mu_{1}+\eta\left(\mu_{2}, \mu_{1}, m\right)\right)$, we have

$$
\begin{aligned}
& \int_{0}^{1}\left|\psi^{\prime}\left(\frac{m \mu_{2}}{c}+\theta \eta\left(\mu_{1}, \frac{\mu_{2}}{c}, m\right)\right)\right|^{q} d \theta \\
& =\left|\psi^{\prime}\left(\mu_{1}\right)\right|^{q} \int_{0}^{1} \frac{1}{n} \sum_{i=1}^{n}(1-s(1-\theta))^{i} d \theta+\left|\psi^{\prime}\left(\frac{\mu_{2}}{c}\right)\right|^{q} \int_{0}^{1} \frac{1}{n} \sum_{i=1}^{n} m^{i}\left(1-(s \theta)^{i}\right) d \theta .
\end{aligned}
$$

Now, Equation (21) becomes

$$
\begin{aligned}
& \left|\frac{\psi\left(\mu_{1}\right)+\psi\left(m \mu_{1}+\eta\left(\frac{\mu_{2}}{c}, \mu_{1}, m\right)\right)}{2}-\frac{c}{\eta\left(\mu_{2}, c \mu_{1}, m\right)} \int_{\mu_{1}}^{m \mu_{1}+\eta\left(\frac{\mu_{2}}{c}, \mu_{1}, m\right)} \psi(x) d x\right| \\
& \leq \frac{\eta\left(\mu_{2}, c \mu_{1}, m\right)}{2 c}\left[\left(\int_{0}^{1}(1-\theta)|1-2 \theta| d \theta\right)^{1-1 / q}\right. \\
& \times\left(\left|\psi^{\prime}\left(\mu_{1}\right)\right|^{q} \int_{0}^{1}(1-\theta)|1-2 \theta| \sum_{i=1}^{n}(1-s(1-\theta))^{i} d \theta\right. \\
& \left.+\left|\psi^{\prime}\left(\frac{\mu_{2}}{c}\right)\right|^{q} \int_{0}^{1}(1-\theta)|1-2 \theta| \frac{1}{n} \sum_{i=1}^{n} m^{i}\left(1-(s \theta)^{i}\right) d \theta\right)^{1 / q} \\
& +\left(\int_{0}^{1} \theta|1-2 \theta| d \theta\right)^{1-1 / q}\left(\left|\psi^{\prime}\left(\mu_{1}\right)\right|^{q} \int_{0}^{1} \theta|1-2 \theta| \frac{1}{n} \sum_{i=1}^{n}(1-s(1-\theta))^{i} d \theta\right. \\
& \left.\left.+\left|\psi^{\prime}\left(\frac{\mu_{2}}{c}\right)\right|^{q} \int_{0}^{1} \theta|1-2 \theta| \frac{1}{n} \sum_{i=1}^{n} m^{i}\left(1-(s \theta)^{i}\right) d \theta\right)^{1 / q}\right] .
\end{aligned}
$$


Since,

$$
\begin{aligned}
& \int_{0}^{1}(1-\theta)|1-2 \theta|(1-s(1-\theta)) d \theta=\int_{0}^{1} \theta|1-2 \theta|(1-s \theta) d \theta=-\frac{3 s-4}{16} . \\
& \int_{0}^{1} \theta|1-2 \theta|(1-s(1-\theta)) d \theta=\int_{0}^{1}(1-\theta)|1-2 \theta|(1-s \theta) d \theta=-\frac{s-4}{16} . \\
& \int_{0}^{1} \theta|1-2 \theta| d \theta=\int_{0}^{1}(1-\theta)|1-2 \theta| d \theta=\left[\frac{1}{4}\right] .
\end{aligned}
$$

Using the computations in (23) gives us the desired result.

Corollary 18. Considering $n=s=1$ in Theorem 15, then

$$
\begin{aligned}
& \left|\frac{\psi\left(\mu_{1}\right)+\psi\left(m \mu_{1}+\eta\left(\frac{\mu_{2}}{c}, \mu_{1}, m\right)\right)}{2}-\frac{c}{\eta\left(\mu_{2}, c \mu_{1}, m\right)} \int_{\mu_{1}}^{m \mu_{1}+\eta\left(\frac{\mu_{2}}{c}, \mu_{1}, m\right)} \psi(x) d x\right| \\
& \leq \frac{\eta\left(\mu_{2}, c \mu_{1}, m\right)}{2 c}\left[\frac{1}{4}\right]^{1-1 / q}\left[\left\{\frac{1}{16}\left|\psi^{\prime}\left(\mu_{1}\right)\right|^{q}+\frac{3 m}{16}\left|\psi^{\prime}\left(\frac{\mu_{2}}{c}\right)\right|^{q}\right\}^{1 / q}\right. \\
& \left.+\left\{\frac{3}{16}\left|\psi^{\prime}\left(\mu_{1}\right)\right|^{q}+\frac{m}{16}\left|\psi^{\prime}\left(\frac{\mu_{2}}{c}\right)\right|^{q}\right\}^{1 / q}\right] .
\end{aligned}
$$

Corollary 19. Considering $n=s=m=1$ in Theorem 15, then

$$
\begin{aligned}
& \left|\frac{\psi\left(\mu_{1}\right)+\psi\left(\mu_{1}+\eta\left(\frac{\mu_{2}}{c}, \mu_{1}\right)\right)}{2}-\frac{c}{\eta\left(\mu_{2}, c \mu_{1}\right)} \int_{\mu_{1}}^{\mu_{1}+\eta\left(\frac{\mu_{2}}{c}, \mu_{1}\right)} \psi(x) d x\right| \\
& \leq \frac{\eta\left(\mu_{2}, c \mu_{1}\right)}{2 c}\left[\frac{1}{4}\right]^{1-1 / q}\left[\left\{\frac{1}{16}\left|\psi^{\prime}\left(\mu_{1}\right)\right|^{q}+\frac{3}{16}\left|\psi^{\prime}\left(\frac{\mu_{2}}{c}\right)\right|^{q}\right\}^{1 / q}\right. \\
& \left.+\left\{\frac{3}{16}\left|\psi^{\prime}\left(\mu_{1}\right)\right|^{q}+\frac{1}{16}\left|\psi^{\prime}\left(\frac{\mu_{2}}{c}\right)\right|^{q}\right\}^{1 / q}\right] .
\end{aligned}
$$

Corollary 20. Considering $n=1$ and $\eta\left(\mu_{1}, \mu_{2}, m\right)=\mu_{1}-m \mu_{2}$ in Theorem 15, then

$$
\begin{aligned}
& \left|\frac{\psi\left(\mu_{1}\right)+\psi\left(\frac{\mu_{2}}{c}\right)}{2}-\frac{c}{\left(\mu_{2}-m c \mu_{1}\right)} \int_{\mu_{1}}^{\frac{\mu_{2}}{c}} \psi(x) d x\right| \\
& \leq \frac{\left(\mu_{2}-m c \mu_{1}\right)}{2 c}\left[\frac{1}{4}\right]^{1-1 / q}\left[\left\{\frac{4-3 s}{16}\left|\psi^{\prime}\left(\mu_{1}\right)\right|^{q}+\frac{4-s}{16} m\left|\psi^{\prime}\left(\frac{\mu_{2}}{c}\right)\right|^{q}\right\}^{1 / q}\right. \\
& \left.+\left\{\frac{4-s}{16}\left|\psi^{\prime}\left(\mu_{1}\right)\right|^{q}+\frac{4-3 s}{16} m\left|\psi^{\prime}\left(\frac{\mu_{2}}{c}\right)\right|^{q}\right\}^{1 / q}\right] .
\end{aligned}
$$

Corollary 21. Considering $n=s=1$ and $\eta\left(\mu_{1}, \mu_{2}, m\right)=\mu_{1}-m \mu_{2}$ in Theorem 15, then

$$
\begin{aligned}
& \left|\frac{\psi\left(\mu_{1}\right)+\psi\left(\frac{\mu_{2}}{c}\right)}{2}-\frac{c}{\left(\mu_{2}-m c \mu_{1}\right)} \int_{\mu_{1}}^{\frac{\mu_{2}}{c}} \psi(x) d x\right| \\
& \leq \frac{\left(\mu_{2}-m c \mu_{1}\right)}{2 c}\left[\frac{1}{4}\right]^{1-1 / q}\left[\left\{\frac{1}{16}\left|\psi^{\prime}\left(\mu_{1}\right)\right|^{q}+\frac{3 m}{16}\left|\psi^{\prime}\left(\frac{\mu_{2}}{c}\right)\right|^{q}\right\}^{1 / q}\right. \\
& \left.+\left\{\frac{3}{16}\left|\psi^{\prime}\left(\mu_{1}\right)\right|^{q}+\frac{m}{16}\left|\psi^{\prime}\left(\frac{\mu_{2}}{c}\right)\right|^{q}\right\}^{1 / q}\right] .
\end{aligned}
$$

Theorem 16. Let $\psi$ be defined as in Theorem 10. If $\left|\psi^{\prime}\right|$ is an n-polynomial s-type m-preinvex function on $\mathbb{X}^{\circ}$ for some fixed $m \in(0,1]$ and $s \in[0,1]$, then for each $x \in\left(m \mu_{1}, m \mu_{1}+\eta\left(\mu_{2}, \mu_{1}, m\right)\right)$. 
If $\left|\psi^{\prime}\right|^{q}$ is an $n$-polynomial s-type $m$-preinvex function on $\mathbb{X}^{\circ}$ for some fixed $m \in(0,1], s \in[0,1]$ and for $q \geq 1$, then for each $x \in\left(m \mu_{1}, m \mu_{1}+\eta\left(\mu_{2}, \mu_{1}, m\right)\right)$, the following inequality holds:

$$
\begin{aligned}
& \left|\frac{\psi\left(\mu_{1}\right)+\psi\left(m \mu_{1}+\eta\left(\frac{\mu_{2}}{c}, \mu_{1}, m\right)\right)}{2}-\frac{c}{\eta\left(\mu_{2}, c \mu_{1}, m\right)} \int_{\mu_{1}}^{m \mu_{1}+\eta\left(\frac{\mu_{2}}{c}, \mu_{1}, m\right)} \psi(x) d x\right| \\
& \leq \frac{\eta\left(\mu_{2}, c \mu_{1}, m\right)}{2 c}\left[\frac{1}{2}\right]^{1-\frac{1}{q}}\left\{\sum_{i=1}^{n}\left(\frac{2-s}{4}\right)^{i}\left[\left|\psi^{\prime}\left(\mu_{1}\right)\right|^{q}+m\left|\psi^{\prime}\left(\frac{\mu_{2}}{c}\right)\right|^{q}\right]\right\}^{1 / q} .
\end{aligned}
$$

Proof. From Lemma 3 and using power-mean inequality, we have

$$
\begin{aligned}
& \left|\frac{\psi\left(\mu_{1}\right)+\psi\left(m \mu_{1}+\eta\left(\frac{\mu_{2}}{c}, \mu_{1}, m\right)\right)}{2}-\frac{c}{\eta\left(\mu_{2}, c \mu_{1}, m\right)} \int_{\mu_{1}}^{m \mu_{1}+\eta\left(\frac{\mu_{2}}{c}, \mu_{1}, m\right)} \psi(x) d x\right| \\
& \leq \frac{\eta\left(\mu_{2}, c \mu_{1}, m\right)}{2 c} \int_{0}^{1}|1-2 \theta|\left|\psi^{\prime}\left(\frac{m \mu_{2}}{c}+\theta \eta\left(\mu_{1}, \frac{\mu_{2}}{c}, m\right)\right)\right| d \theta \\
& \leq \frac{\eta\left(\mu_{2}, c \mu_{1}, m\right)}{2 c}\left(\int_{0}^{1}|1-2 \theta| d \theta\right)^{1-1 / q}\left(\int_{0}^{1}|1-2 \theta|\left|\psi^{\prime}\left(\frac{m \mu_{2}}{c}+\theta \eta\left(\mu_{1}, \frac{\mu_{2}}{c}, m\right)\right)\right|{ }^{q} d \theta\right)^{1 / q} .
\end{aligned}
$$

Since $\left|\psi^{\prime}\right|^{q}$ is an $n$-polynomial s-type $m$-preinvex on $\left(\mu_{1}, m \mu_{1}+\eta\left(\mu_{2}, \mu_{1}, m\right)\right)$, we have

$$
\begin{aligned}
& \int_{0}^{1}\left|\psi^{\prime}\left(\frac{m \mu_{2}}{c}+\theta \eta\left(\mu_{1}, \frac{\mu_{2}}{c}, m\right)\right)\right|^{q} d \theta \\
& =\left|\psi^{\prime}\left(\mu_{1}\right)\right|^{q} \int_{0}^{1} \sum_{i=1}^{n}(1-s(1-\theta))^{i} d \theta+\left|\psi^{\prime}\left(\frac{\mu_{2}}{c}\right)\right|^{q} \int_{0}^{1} \sum_{i=1}^{n}\left(1-(s \theta)^{i}\right) d \theta .
\end{aligned}
$$

Now, Equation (25) becomes

$$
\begin{aligned}
& \left|\frac{\psi\left(\mu_{1}\right)+\psi\left(m \mu_{1}+\eta\left(\frac{\mu_{2}}{c}, \mu_{1}, m\right)\right)}{2}-\frac{c}{\eta\left(\mu_{2}, c \mu_{1}, m\right)} \int_{\mu_{1}}^{\mu_{1}+\eta\left(\frac{m \mu_{2}}{c}, \mu_{1}, m\right)} \psi(x) d x\right| \\
& \leq \frac{\eta\left(\mu_{2}, c \mu_{1}, m\right)}{2 c}\left(\int_{0}^{1}|1-2 \theta| d \theta\right)^{1-1 / q}\left(\left|\psi^{\prime}\left(\mu_{1}\right)\right|^{q} \int_{0}^{1}|1-2 \theta| \sum_{i=1}^{n}(1-s(1-\theta))^{i} d \theta\right. \\
& \left.+\left|\psi^{\prime}\left(\frac{\mu_{2}}{c}\right)\right|^{q} \int_{0}^{1}|1-2 \theta| \sum_{i=1}^{n} m^{i}\left(1-(s \theta)^{i}\right) d \theta\right)^{1 / q} .
\end{aligned}
$$

Since,

$$
\begin{aligned}
& \int_{0}^{1}|1-2 \theta|(1-s(1-\theta)) d \theta=\int_{0}^{1}|1-2 \theta|(1-s \theta) d \theta=-\frac{s-2}{4} . \\
& \int_{0}^{1}|1-2 \theta| d \theta=\frac{1}{2} .
\end{aligned}
$$

The proof of the Theorem gets completed by using these computations in (26).

Corollary 22. Considering $n=s=1$ in Theorem 16, we obtain the following inequality:

$$
\begin{aligned}
& \left|\frac{\psi\left(\mu_{1}\right)+\psi\left(m \mu_{1}+\eta\left(\frac{\mu_{2}}{c}, \mu_{1}, m\right)\right)}{2}-\frac{c}{\eta\left(\mu_{2}, c \mu_{1}, m\right)} \int_{\mu_{1}}^{m \mu_{1}+\eta\left(\frac{\mu_{2}}{c}, \mu_{1}, m\right)} \psi(x) d x\right| \\
& \leq \frac{\eta\left(\mu_{2}, c \mu_{1}, m\right)}{2^{\frac{2 q+1}{q}} c}\left\{\left[\left|\psi^{\prime}\left(\mu_{1}\right)\right|^{q}+m\left|\psi^{\prime}\left(\frac{\mu_{2}}{c}\right)\right|^{q}\right]\right\}^{1 / q} .
\end{aligned}
$$


Corollary 23. Considering $n=s=m=1$ in Theorem 16, we obtain the following inequality:

$$
\begin{aligned}
& \left|\frac{\psi\left(\mu_{1}\right)+\psi\left(\mu_{1}+\eta\left(\frac{\mu_{2}}{c}, \mu_{1}\right)\right)}{2}-\frac{c}{\eta\left(\mu_{2}, c \mu_{1}\right)} \int_{\mu_{1}}^{\mu_{1}+\eta\left(\frac{\mu_{2}}{c}, \mu_{1}\right)} \psi(x) d x\right| \\
& \leq \frac{\eta\left(\mu_{2}, c \mu_{1}\right)}{2^{\frac{2 q+1}{q}} c}\left\{\left[\left|\psi^{\prime}\left(\mu_{1}\right)\right|^{q}+\left|\psi^{\prime}\left(\frac{\mu_{2}}{c}\right)\right|^{q}\right]\right\}^{1 / q} .
\end{aligned}
$$

Corollary 24. If we set $n=1$ and $\eta\left(\mu_{1}, \mu_{2}, m\right)=\mu_{1}-m \mu_{2}$ in Theorem 16 , then

$$
\begin{aligned}
& \left|\frac{\psi\left(\mu_{1}\right)+\psi\left(\frac{\mu_{2}}{c}\right)}{2}-\frac{c}{\left(\mu_{2}-m c \mu_{1}\right)} \int_{\mu_{1}}^{\frac{\mu_{2}}{c}} \psi(x) d x\right| \\
& \leq \frac{\left(\mu_{2}-m c \mu_{1}\right)}{2 c}\left[\frac{1}{2}\right]^{1-\frac{1}{q}}\left\{\frac{2-s}{4}\left[\left|\psi^{\prime}\left(\mu_{1}\right)\right|^{q}+m\left|\psi^{\prime}\left(\frac{\mu_{2}}{c}\right)\right|^{q}\right]\right\}^{1 / q} .
\end{aligned}
$$

Corollary 25. If we set $n=s=1$ and $\eta\left(\mu_{1}, \mu_{2}, m\right)=\mu_{1}-m \mu_{2}$, in Theorem 16, then

$$
\begin{aligned}
& \left|\frac{\psi\left(\mu_{1}\right)+\psi\left(\frac{\mu_{2}}{c}\right)}{2}-\frac{c}{\left(\mu_{2}-m c \mu_{1}\right)} \int_{\mu_{1}}^{\frac{\mu_{2}}{c}} \psi(x) d x\right| \\
& \leq \frac{\left(\mu_{2}-m c \mu_{1}\right)}{2^{\frac{2 q+1}{q}} c}\left\{\left[\left|\psi^{\prime}\left(\mu_{1}\right)\right|^{q}+m\left|\psi^{\prime}\left(\frac{\mu_{2}}{c}\right)\right|^{q}\right]\right\}^{1 / q} .
\end{aligned}
$$

Theorem 17. Let $\psi$ be defined as in Theorem 10. If $\left|\psi^{\prime}\right|$ is an n-polynomial s-type m-preinvex function on $\mathbb{X}^{\circ}$ for some fixed $m \in(0,1]$ and $s \in[0,1]$, then for each $x \in\left(m \mu_{1}, m \mu_{1}+\eta\left(\mu_{2}, \mu_{1}, m\right)\right)$. If $\left|\psi^{\prime}\right|^{q}$ is an n-polynomial s-type m-preinvex function on $\mathbb{X}^{\circ}$ for some fixed $m \in(0,1], s \in[0,1]$ and for $p, q>1, \frac{1}{q}+\frac{1}{p}=1$, then for each $x \in\left(m \mu_{1}, m \mu_{1}+\eta\left(\mu_{2}, \mu_{1}, m\right)\right)$, the following inequality holds:

$$
\begin{aligned}
& \left|\frac{c}{\eta\left(\mu_{2}, c \mu_{1}, m\right)} \int_{\mu_{1}}^{m \mu_{1}+\eta\left(\frac{\mu_{2}}{c}, \mu_{1}, m\right)} \psi(x) d x-\psi\left(\frac{2 m \mu_{1}+\eta\left(\mu_{2}, \mu_{1}, m\right)}{2 c}\right)\right| \\
& \leq \frac{\eta\left(\mu_{2}, c \mu_{1}, m\right)}{c}\left[\left(\frac{1}{p+1}\right)^{1 / p}\left\{\sum_{i=1}^{n}\left(\frac{2-s}{2}\right)^{i}\left[\left|\psi^{\prime}\left(\mu_{1}\right)\right|^{q}+m^{i}\left|\psi^{\prime}\left(\frac{\mu_{2}}{c}\right)\right|^{q}\right]\right\}^{1 / q}\right. \\
& \left.+\left\{\sum_{i=1}^{n}\left(\frac{4-3 s}{8}\right)^{i}\left[\left|\psi^{\prime}\left(\mu_{1}\right)\right|^{q}+m^{i}\left|\psi^{\prime}\left(\frac{\mu_{2}}{c}\right)\right|^{q}\right]\right\}^{1 / q}\right] .
\end{aligned}
$$

Proof. From Lemma 4 and using Hölder's inequality, we have

$$
\begin{aligned}
& \left|\frac{c}{\eta\left(\mu_{2}, c \mu_{1}, m\right)} \int_{\mu_{1}}^{m \mu_{1}+\eta\left(\frac{\mu_{2}}{c}, \mu_{1}, m\right)} \psi(x) d x-\psi\left(\frac{2 m \mu_{1}+\eta\left(\mu_{2}, \mu_{1}, m\right)}{2 c}\right)\right| \\
& =\frac{\eta\left(\mu_{2}, c \mu_{1}, m\right)}{c}\left\{\int_{0}^{1} \theta \psi^{\prime}\left(\frac{m \mu_{2}}{c}+\theta \eta\left(\mu_{1}, \frac{\mu_{2}}{c}, m\right)\right) d \theta-\int_{1 / 2}^{1} \psi^{\prime}\left(\frac{m \mu_{2}}{c}+\theta \eta\left(\mu_{1}, \frac{\mu_{2}}{c}, m\right)\right) d \theta\right\} \\
& \leq \frac{\eta\left(\mu_{2}, c \mu_{1}, m\right)}{c}\left[\left(\int_{0}^{1} \theta^{p} d \theta\right)^{1 / p}\left\{\int_{0}^{1}\left|\psi^{\prime}\left(\frac{m \mu_{2}}{c}+\theta \eta\left(\mu_{1}, \frac{\mu_{2}}{c}, m\right)\right)\right|^{q} d \theta\right\}^{1 / q}\right. \\
& \left.+\left\{\int_{1 / 2}^{1}\left|\psi^{\prime}\left(\frac{m \mu_{2}}{c}+\theta \eta\left(\mu_{1}, \frac{\mu_{2}}{c}, m\right)\right)\right|^{q} d \theta\right\}^{1 / q}\right]
\end{aligned}
$$




$$
\begin{aligned}
& \leq \frac{\eta\left(\mu_{2}, c \mu_{1}, m\right)}{c}\left[( \frac { 1 } { p + 1 } ) ^ { 1 / p } \left\{\int_{0}^{1} \sum_{i=1}^{n}(1-s(1-\theta))\left|\psi^{\prime}\left(\mu_{1}\right)\right|^{q} d \theta\right.\right. \\
& \left.+\int_{0}^{1} \sum_{i=1}^{n} m^{i}\left(1-(s \theta)^{i}\right)\left|\psi^{\prime}\left(\frac{\mu_{2}}{c}\right)\right|{ }^{q} d \theta\right\}^{1 / q} \\
& +\left\{\int_{1 / 2}^{1} \sum_{i=1}^{n}\left(1-s(1-\theta)^{i}\left|\psi^{\prime}\left(\mu_{1}\right)\right|^{q} d \theta+\int_{1 / 2}^{1} \sum_{i=1}^{n} m^{i}\left(1-(s \theta)^{i}\right)\left|\psi^{\prime}\left(\frac{\mu_{2}}{c}\right)\right|^{q} d \theta\right\}^{1 / q}\right] \\
& =\frac{\eta\left(\mu_{2}, c \mu_{1}, m\right)}{c}\left[\left(\frac{1}{p+1}\right)^{1 / p}\left\{\sum_{i=1}^{n}\left(\frac{2-s}{2}\right)^{i}\left[\left|\psi^{\prime}\left(\mu_{1}\right)\right|^{q}+m^{i}\left|\psi^{\prime}\left(\frac{\mu_{2}}{c}\right)\right|^{q}\right]\right\}^{1 / q}\right. \\
& \left.+\left\{\sum_{i=1}^{n}\left(\frac{4-3 s}{8}\right)^{i}\left[\left|\psi^{\prime}\left(\mu_{1}\right)\right|^{q}+m^{i}\left|\psi^{\prime}\left(\frac{\mu_{2}}{c}\right)\right|^{q}\right]\right\}^{1 / q}\right] .
\end{aligned}
$$

Corollary 26. Cobsidering $n=s=1$ in Theorem 17, we obtain the following inequality:

$$
\begin{aligned}
& \left|\frac{c}{\eta\left(\mu_{2}, c \mu_{1}, m\right)} \int_{\mu_{1}}^{m \mu_{1}+\eta\left(\frac{\mu_{2}}{c}, \mu_{1}, m\right)} \psi(x) d x-\psi\left(\frac{2 m \mu_{1}+\eta\left(\mu_{2}, \mu_{1}, m\right)}{2 c}\right)\right| \\
& \leq \frac{\eta\left(\mu_{2}, c \mu_{1}, m\right)}{c}\left[\left(\frac{1}{p+1}\right)^{1 / p}\left\{\frac{1}{2}\left[\left|\psi^{\prime}\left(\mu_{1}\right)\right|^{q}+m\left|\psi^{\prime}\left(\frac{\mu_{2}}{c}\right)\right|^{q}\right]\right\}^{1 / q}\right. \\
& \left.+\left\{\frac{1}{8}\left[\left|\psi^{\prime}\left(\mu_{1}\right)\right|^{q}+m\left|\psi^{\prime}\left(\frac{\mu_{2}}{c}\right)\right|^{q}\right]\right\}^{1 / q}\right] .
\end{aligned}
$$

Corollary 27. Considering $n=s=m=1$ in Theorem 17, then

$$
\begin{aligned}
& \left|\frac{c}{\eta\left(\mu_{2}, c \mu_{1}\right)} \int_{\mu_{1}}^{\mu_{1}+\eta\left(\frac{\mu_{2}}{c}, \mu_{1}\right)} \psi(x) d x-\psi\left(\frac{2 \mu_{1}+\eta\left(\mu_{2}, \mu_{1}\right)}{2 c}\right)\right| \\
& \leq \frac{\eta\left(\mu_{2}, c \mu_{1}\right)}{c}\left[\left(\frac{1}{p+1}\right)^{1 / p}\left\{\frac{1}{2}\left[\left|\psi^{\prime}\left(\mu_{1}\right)\right|^{q}+\left|\psi^{\prime}\left(\frac{\mu_{2}}{c}\right)\right|^{q}\right]\right\}^{1 / q}+\left\{\frac{1}{8}\left[\left|\psi^{\prime}\left(\mu_{1}\right)\right|^{q}+\left|\psi^{\prime}\left(\frac{\mu_{2}}{c}\right)\right|^{q}\right]\right\}^{1 / q}\right] .
\end{aligned}
$$

Corollary 28. If we set $n=1$ and $\eta\left(\mu_{1}, \mu_{2}, m\right)=\mu_{1}-m \mu_{2}$ in Theorem 17 , then

$$
\begin{aligned}
& \left|\frac{c}{\left(\mu_{2}-m c \mu_{1}\right)} \int_{\mu_{1}}^{\frac{\mu_{2}}{c}} \psi(x) d x-\psi\left(\frac{m \mu_{1}+\mu_{2}}{2 c}\right)\right| \\
& \leq \frac{\left(\mu_{2}-m c \mu_{1}\right)}{c}\left[\left(\frac{1}{p+1}\right)^{1 / p}\left\{\frac{2-s}{2}\left[\left|\psi^{\prime}\left(\mu_{1}\right)\right|^{q}+m\left|\psi^{\prime}\left(\frac{\mu_{2}}{c}\right)\right|^{q}\right]\right\}^{1 / q}\right. \\
& \left.+\left\{\frac{4-3 s}{8}\left[\left|\psi^{\prime}\left(\mu_{1}\right)\right|^{q}+m\left|\psi^{\prime}\left(\frac{\mu_{2}}{c}\right)\right|^{q}\right]\right\}^{1 / q}\right] .
\end{aligned}
$$


Corollary 29. If we set $n=s=1$ and $\eta\left(\mu_{1}, \mu_{2}, m\right)=\mu_{1}-m \mu_{2}$ in Theorem 17 , then

$$
\begin{aligned}
& \left|\frac{c}{\left(\mu_{2}-m c \mu_{1}\right)} \int_{\mu_{1}}^{\frac{\mu_{2}}{c}} \psi(x) d x-\psi\left(\frac{m \mu_{1}+\mu_{2}}{2 c}\right)\right| \\
& \leq \frac{\left(\mu_{2}-m c \mu_{1}\right)}{c}\left[\left(\frac{1}{p+1}\right)^{1 / p}\left\{\frac{1}{2}\left[\left|\psi^{\prime}\left(\mu_{1}\right)\right|^{q}+m\left|\psi^{\prime}\left(\frac{\mu_{2}}{c}\right)\right|^{q}\right]\right\}^{1 / q}\right. \\
& \left.+\left\{\frac{1}{8}\left[\left|\psi^{\prime}\left(\mu_{1}\right)\right| q+m\left|\psi^{\prime}\left(\frac{\mu_{2}}{c}\right)\right|^{q}\right]\right\}^{1 / q}\right] .
\end{aligned}
$$

Theorem 18. Let $\psi$ be defined as in Theorem 10. If $\left|\psi^{\prime}\right|$ is an n-polynomial s-type m-preinvex function on $\mathbb{X}^{\circ}$ for some fixed $m \in(0,1]$ and $s \in[0,1]$, then for each $x \in\left(m \mu_{1}, m \mu_{1}+\eta\left(\mu_{2}, \mu_{1}, m\right)\right)$. If $\left|\psi^{\prime}\right|^{q}$ is an $n$-polynomial s-type $m$-preinvex function on $\mathbb{X}^{\circ}$ for some fixed $m \in(0,1], s \in[0,1]$ and for $q \geq 1$, then for each $x \in\left(m \mu_{1}, m \mu_{1}+\eta\left(\mu_{2}, \mu_{1}, m\right)\right)$, the following inequality holds:

$$
\begin{aligned}
& \left|\frac{c}{\eta\left(\mu_{2}, c \mu_{1}, m\right)} \int_{\mu_{1}}^{m \mu_{1}+\eta\left(\frac{\mu_{2}}{c}, \mu_{1}, m\right)} \psi(x) d x-\psi\left(\frac{2 m \mu_{1}+\eta\left(\mu_{2}, \mu_{1}, m\right)}{2 c}\right)\right| \\
& \leq \frac{\eta\left(\mu_{2}, c \mu_{1}, m\right)}{c}\left[\left(\frac{1}{2}\right)^{1-1 / q}\left\{\sum_{i=1}^{n}\left(\frac{3-s}{6}\right)\left|\psi^{\prime}\left(\mu_{1}\right)\right|^{q}+\sum_{i=1}^{n}\left(\frac{3-2 s}{6}\right) m^{i}\left|\psi^{\prime}\left(\frac{\mu_{2}}{c}\right)\right|^{q}\right\}^{1 / q}\right. \\
& \left.+\left\{\sum_{i=1}^{n}\left(\frac{4-3 s}{8}\right)^{i}\left[\left|\psi^{\prime}\left(\mu_{1}\right)\right|^{q}+m^{i}\left|\psi^{\prime}\left(\frac{\mu_{2}}{c}\right)\right|^{q}\right]\right\}^{1 / q}\right] .
\end{aligned}
$$

Proof. From Lemma 4 and using power-mean inequality, we have

$$
\begin{aligned}
& \left|\frac{c}{\eta\left(\mu_{2}, c \mu_{1}, m\right)} \int_{\mu_{1}}^{m \mu_{1}+\eta\left(\frac{\mu_{2}}{c}, \mu_{1}, m\right)} \psi(x) d x-\psi\left(\frac{2 m \mu_{1}+\eta\left(\mu_{2}, \mu_{1}, m\right)}{2 c}\right)\right| \\
& =\frac{\eta\left(\mu_{2}, c \mu_{1}, m\right)}{c}\left\{\int_{0}^{1} \theta \psi^{\prime}\left(\frac{m \mu_{2}}{c}+\theta \eta\left(\mu_{1}, \frac{\mu_{2}}{c}, m\right)\right) d \theta-\int_{1 / 2}^{1} \psi^{\prime}\left(\frac{m \mu_{2}}{c}+\theta \eta\left(\mu_{1}, \frac{\mu_{2}}{c}, m\right)\right) d \theta\right\} \\
& \leq \frac{\eta\left(\mu_{2}, c \mu_{1}, m\right)}{c}\left[\left(\int_{0}^{1} \theta d \theta\right)^{1-1 / q}\left\{\int_{0}^{1} \theta\left|\psi^{\prime}\left(\frac{m \mu_{2}}{c}+\theta \eta\left(\mu_{1}, \frac{\mu_{2}}{c}, m\right)\right)\right|{ }^{q} d \theta\right\}^{1 / q}\right. \\
& \left.+\left\{\int_{1 / 2}^{1}\left|\psi^{\prime}\left(\frac{m \mu_{2}}{c}+\theta \eta\left(\mu_{1}, \frac{\mu_{2}}{c}, m\right)\right)\right|^{q} d \theta\right\}^{1 / q}\right] \\
& \leq \frac{\eta\left(\mu_{2}, c \mu_{1}, m\right)}{c}\left[( \frac { 1 } { 2 } ) ^ { 1 - 1 / q } \left\{\int_{0}^{1} \theta \sum_{i=1}^{n}(1-s(1-\theta))^{i}\left|\psi^{\prime}\left(\mu_{1}\right)\right|^{q} d \theta\right.\right. \\
& \left.+\int_{0}^{1} \theta \sum_{i=1}^{n} m^{i}\left(1-(s \theta)^{i}\right)\left|\psi^{\prime}\left(\frac{\mu_{2}}{c}\right)\right|^{q} d \theta\right\}^{1 / q} \\
& \left.+\left\{\int_{1 / 2}^{1} \sum_{i=1}^{n}(1-s(1-\theta))^{i}\left|\psi^{\prime}\left(\mu_{1}\right)\right|^{q} d \theta+\int_{1 / 2}^{1} \sum_{i=1}^{n} m^{i}\left(1-(s \theta)^{i}\right)\left|\psi^{\prime}\left(\frac{\mu_{2}}{c}\right)\right|{ }^{q} d \theta\right\}^{1 / q}\right] \\
& =\frac{\eta\left(\mu_{2}, c \mu_{1}, m\right)}{c}\left[\left(\frac{1}{2}\right)^{1-1 / q}\left\{\sum_{i=1}^{n}\left(\frac{3-s}{6}\right)^{i}\left|\psi^{\prime}\left(\mu_{1}\right)\right|^{q}+\sum_{i=1}^{n}\left(\frac{3-2 s}{6}\right)^{i} m^{i}\left|\psi^{\prime}\left(\frac{\mu_{2}}{c}\right)\right|^{q}\right\}^{1 / q}\right. \\
& \left.+\left\{\sum_{i=1}^{n}\left(\frac{4-3 s}{8}\right)^{i}\left[\left|\psi^{\prime}\left(\mu_{1}\right)\right|^{q}+m^{i}\left|\psi^{\prime}\left(\frac{\mu_{2}}{c}\right)\right|^{q}\right]\right\}^{1 / q}\right] \text {. }
\end{aligned}
$$


Corollary 30. Considering $n=s=1$ in Theorem 18, then

$$
\begin{aligned}
& \left|\frac{c}{\eta\left(\mu_{2}, c \mu_{1}, m\right)} \int_{\mu_{1}}^{m \mu_{1}+\eta\left(\frac{\mu_{2}}{c}, \mu_{1}, m\right)} \psi(x) d x-\psi\left(\frac{2 m \mu_{1}+\eta\left(\mu_{2}, \mu_{1}, m\right)}{2 c}\right)\right| \\
& \leq \frac{\eta\left(\mu_{2}, c \mu_{1}, m\right)}{c}\left[\left(\frac{1}{2}\right)^{1-1 / q}\left\{\frac{1}{3}\left|\psi^{\prime}\left(\mu_{1}\right)\right|^{q}+\frac{m}{6}\left|\psi^{\prime}\left(\frac{\mu_{2}}{c}\right)\right|^{q}\right\}^{1 / q}\right. \\
& \left.+\left\{\frac{1}{8}\left[\left|\psi^{\prime}\left(\mu_{1}\right)\right|^{q}+m\left|\psi^{\prime}\left(\frac{\mu_{2}}{c}\right)\right|^{q}\right]\right\}^{1 / q}\right] .
\end{aligned}
$$

Corollary 31. Considering $n=s=m=1$ in Theorem 18 , then

$$
\begin{aligned}
& \left|\frac{c}{\eta\left(\mu_{2}, c \mu_{1}\right)} \int_{\mu_{1}}^{\mu_{1}+\eta\left(\frac{\mu_{2}}{c}, \mu_{1}\right)} \psi(x) d x-\psi\left(\frac{2 \mu_{1}+\eta\left(\mu_{2}, \mu_{1}\right)}{2 c}\right)\right| \\
& \leq \frac{\eta\left(\mu_{2}, c \mu_{1}\right)}{c}\left[\left(\frac{1}{2}\right)^{1-1 / q}\left\{\frac{1}{3}\left|\psi^{\prime}\left(\mu_{1}\right)\right|^{q}+\frac{1}{6}\left|\psi^{\prime}\left(\frac{\mu_{2}}{c}\right)\right|^{q}\right\}^{1 / q}+\left\{\frac{1}{8}\left[\left|\psi^{\prime}\left(\mu_{1}\right)\right|^{q}+\left|\psi^{\prime}\left(\frac{\mu_{2}}{c}\right)\right|^{q}\right]\right\}^{1 / q}\right] .
\end{aligned}
$$

Corollary 32. Considering $n=1$ and $\eta\left(\mu_{1}, \mu_{2}, m\right)=\mu_{1}-m \mu_{2}$ in Theorem 18, then

$$
\begin{aligned}
& \left|\frac{c}{\left(\mu_{2}-m c \mu_{1}\right)} \int_{\mu_{1}}^{\frac{\mu_{2}}{c}} \psi(x) d x-\psi\left(\frac{\left.m \mu_{1}+\mu_{2}\right)}{2 c}\right)\right| \\
& \leq \frac{\left(\mu_{2}-m c \mu_{1}\right)}{c}\left[\left(\frac{1}{2}\right)^{1-1 / q}\left\{\frac{3-s}{6}\left|\psi^{\prime}\left(\mu_{1}\right)\right|^{q}+\frac{3-2 s}{6} m\left|\psi^{\prime}\left(\frac{\mu_{2}}{c}\right)\right|^{q}\right\}^{1 / q}\right. \\
& \left.+\left\{\frac{4-3 s}{8}\left[\left|\psi^{\prime}\left(\mu_{1}\right)\right|^{q}+m\left|\psi^{\prime}\left(\frac{\mu_{2}}{c}\right)\right|^{q}\right]\right\}^{1 / q}\right] .
\end{aligned}
$$

Corollary 33. If we set $n=s=1$ and $\eta\left(\mu_{1}, \mu_{2}, m\right)=\mu_{1}-m \mu_{2}$ in Theorem 18, then

$$
\begin{aligned}
& \left|\frac{c}{\left(\mu_{2}-m c \mu_{1}\right)} \int_{\mu_{1}}^{\frac{\mu_{2}}{c}} \psi(x) d x-\psi\left(\frac{m \mu_{1}+\mu_{2}}{2 c}\right)\right| \\
& \leq \frac{\left(\mu_{2}-m c \mu_{1}\right)}{c}\left[\left(\frac{1}{2}\right)^{1-1 / q}\left\{\frac{1}{3}\left|\psi^{\prime}\left(\mu_{1}\right)\right|^{q}+\frac{m}{6}\left|\psi^{\prime}\left(\frac{\mu_{2}}{c}\right)\right|^{q}\right\}^{1 / q}\right. \\
& \left.+\left\{\frac{1}{8}\left[\left|\psi^{\prime}\left(\mu_{1}\right)\right|^{q}+m\left|\psi^{\prime}\left(\frac{\mu_{2}}{c}\right)\right|^{q}\right]\right\}^{1 / q}\right] .
\end{aligned}
$$

In future, we believe the outcomes of this manuscript and the newly investigated concept can be estimated and generalized in various ways such as time scale calculus, Raina convexity, s-type Raina convexity, fractional calculus and quantam calculus, etc. We believe the procedure and fruitful techniques of this manuscript will motivate for further research in this direction.

\section{Conclusions}

In this article, first we introduced a new notion of preinvexity namely an $n$-polynomial $s$-type $m$-preinvex function and discussed its algebraic properties. Then, we establish a new variant of Hermite-Hadamard type inequality in the setting of the new notion. Next, two new integral equalities are presented for differentiable preinvex function and considering them as auxiliary results, several refinements of Hermite-Hadamard type inequality are reviewed. Employing Lemma 3, we presented three Theorems 12-14, where the Hölder and Hölder-İscan inequality are used. It is observed that Theorem 14 provides better result as compared to Theorems 12 and 13. Similarly, employing Lemma 3, Theorems 15 and 16 
are derived using power-mean and improved power-mean inequality, and we conclude that Theorem 15 provides better result than Theorem 16.

Author Contributions: Conceptualization, M.T. and S.K.S.; methodology, M.T.; H.A.; H.B. and S.K.S.; software, M.T. and S.K.S.; validation, H.A.; H.B.; T.S. and J.R.; formal analysis, M.T.; H.A.; H.B.; S.K.S.; T.S. and J.R.; investigation, M.T.; H.A.; H.B. and S.K.S.; resources, M.T.; H.A. and S.K.S.; data curation, H.B.; T.S. and J.R.; writing—original draft preparation, M.T.; H.A. and S.K.S.; writing-review and editing, M.T. and S.K.S.; supervision, H.A.; H.B.; T.S. and J.R.; project administration, M.T.; H.A. and S.K.S.; funding acquisition, T.S. and J.R. All authors have read and agreed to the published version of the manuscript.

Funding: This research was funded by National Science, Research and Innovation Fund (NSRF), and King Mongkut's University of Technology North Bangkok with Contract no. KMUTNB-FF-65-49.

Institutional Review Board Statement: Not applicable.

Informed Consent Statement: Not applicable.

Acknowledgments: We would like to thank the National Science, Research and Innovation Fund (NSRF), and King Mongkut's University of Technology North Bangkok with Contract no. KMUTNB-FF-65-49.

Conflicts of Interest: The authors declare no conflict of interest.

\section{References}

1. Hardy, G.H.; Little, J.E.; Pólya, G. Inequalities; Cambridge Mathematical Library, Cambridge University Press: Cambridge, UK, 1952.

2. Xi, B.Y.; Qi, F. Some integral inequalities of Hermite-Hadamard type for convex functions with applications to means. J. Funct. Spaces Appl. 2012, 2012, 980438. [CrossRef]

3. Niculescu, C.P.; Persson, L.E. Convex Functions and Their Applications; Springer: New York, NY, USA, 2006.

4. Özcan, S.; İşcan, İ. Some new Hermite-Hadamard type integral inequalities for the $s$-convex functions and theirs applications. J. Inequal. Appl. 2019, 2019, 201. [CrossRef]

5. Khan, M.A.; Chu, Y.M.; Khan, T.U.; Khan, J. Some new inequalities of Hermite-Hadamard type for s-convex functions with applications. Open Math. 2017, 15, 1414-1430. [CrossRef]

6. Tariq, M.; Sahoo, S.K.; Jarad, F.; Kodamasingh, B. Some integral inequalities for generalized preinvex functions with applications. AIMS Math. 2021, 6, 13907-13930. [CrossRef]

7. Tariq, M.; Shaikh, A.A.; Sahoo, S.K.; Ahmad, H.; Sitthiwirattham, T.; Reunsumrit, J. New Integral Inequalities via Generalized Preinvex Functions. Axioms 2021, 10, 296. [CrossRef]

8. Latif, M.A. Hermite-Hadamard-type inequalities for geometrically r-convex functions in terms of Stolarskys mean with applications to means. Adv. Differ. Equ. 2021, 2021, 371. [CrossRef]

9. Hadamard, J. Étude sur les propriétés des fonctions entiéres en particulier d'une fonction considéréé par Riemann. J. Math. Pures. Appl. 1893, 58, 171-215.

10. Baleanu, D.; Purohit, S.D. Chebyshev type integral inequalities involving the fractional hypergeometric operators. Abstr. Appl. Anal. 2014, 2014, 609160. [CrossRef]

11. Guzmán, P.M.; Kórus, P.; Valdés, J.E.N. Generalized integral inequalities of Chebyshev type. Fractal Fract. 2020, 4, 10. [CrossRef]

12. İşcan, İ.; Turhan, S.; Maden, S. Hermite-Hadamard and simpson-like type inequalities for differentiable $p$-quasi convex Functions. Filomat 2017, 31, 5945-5953. [CrossRef]

13. Toader, G. Some generalizations of the convexity. In Proceedings of the Colloquium on Approximation and Optimization, Cluj-Napoca, Romania, 25-27 October 1984; pp. 329-338.

14. Latif, M.A.; Shoaib, M. Hermite-Hadamard type integral inequalities for differentiable $m$-preinvex and $(\alpha, m)-$ preinvex functions. J. Egypt. Math. Soc. 2015, 23, 236-241. [CrossRef]

15. Deng, Y.; Kalsoom, H.; Wu, S. Some new Quantum Hermite-Hadamard-type estimates within a class of generalized ( $s, m)-$ preinvex functions. Symmetry 2019, 11, 1283. [CrossRef]

16. Toplu, T.; Kadakal, M.; İşcan, İ. On n-polynomial convexity and some relatd inequalities. AIMS Math 2020, 5, 1304-1318. [CrossRef]

17. Butt, S.I.; Kashuri, A.; Tariq, M.; Nasir, J.; Aslam, A.; Geo, W. Hermite-Hadamard-type inequalities via n-polynomial exponentialtype convexity and their applications. Adv. Differ. Equ. 2020, 2020, 508. [CrossRef]

18. Butt, S.I.; Tariq, M.; Aslam, A.; Ahmad, H.; Nofel, T.A. Hermite-Hadamard type inequalities via generalized harmonic exponential convexity. J. Funct. Spaces 2021, 2021, 5533491. [CrossRef]

19. Tariq, M.; Nasir, J.; Sahoo, S.K.; Mallah, A.A. A note on some Ostrowski type inequalities via generalized exponentially convex functions. J. Math. Anal. Model. 2021, 2, 1-15. 
20. Rashid, S.; İşcan, İ.; Baleanu, D.; Chu, Y.M. Generation of new fractional inequalities via n-polynomials $s$-type convexity with applications. Adv. Differ. Equ. 2020, 2020, 264. [CrossRef]

21. Park, C.; Chu, Y.M.; Saleem, M.S.; Jahangir, N.; Rehman, N. On n-polynomial p-convex functions and some related inequalities. Adv. Differ. Equ. 2020, 2020, 666. [CrossRef]

22. Chen, S.B.; Rashid, S.; Noor, M.A.; Hammouch, Z.; Chu, Y.M. New fractional approaches for n-polynomial P-convexity with applications in special function theory. Adv. Differ. Equ. 2020, 2020, 543. [CrossRef]

23. Awan, M.U.; Akhtar, N.; Iftikhar, S.; Noor, M.A.; Chu, Y.M. New Hermite-Hadamard type inequalities for n-polynomial harmonically convex functions. J. Inequal. Appl. 2020, 2020, 125. [CrossRef]

24. Du, T.T.; Liao, J.G.; Li, Y.J. Properties and integral inequalities of Hadamard-Simpson type for the generalized $(s, m)-$ preinvex functions. J. Nonlinear Sci. Appl. 2016, 9, 3112-3126. [CrossRef]

25. Weir, T.; Mond, B. Pre-inven functions in multiple objective optimization. J. Math. Anal. Appl. 1988, 136, 29-38. [CrossRef]

26. Noor, M.A. Hadamard integral inequalities for product of two preinvex function. Nonl. Anal. Forum 2009, 14, 167-173.

27. Noor, M.A. Some new classes of nonconvex functions. Nonl. Funct. Anal. Appl. 2006, 11, 165-171.

28. Noor, M.A. On Hadamard integral inequalities invoving two log-preinvex functions. J. Inequal. Pure Appl. Math. $2007,8,75$.

29. Awan, M.U.; Talib, S.; Noor, M.A.; Chu, Y.M.; Noor, K.I. Some trapezium-like inequalities involving functions having strongly n-polynomial preinvexity property of higher order. J. Funct. Spaces 2020, 2020, 9154139. [CrossRef]

30. Mohan, S.R.; Neogy, S.K. On invex sets and preinvex functions. J. Math. Anal. Appl. 1995, 189, 901-908. [CrossRef]

31. Du, T.S.; Liao, J.G.; Chen, L.G.; Awan, M.U. Properties and Riemann-Liouville fractional Hermite-Hadamard inequalities for the generalized $(\alpha, m)$-preinvex functions. J. Inequal. Appl. 2016, 2016, 306. [CrossRef]

32. Mishra, S.K.; Giorgi, G. Invexity and Optimization; Springer: Berlin, Germany, 2008. 